\title{
Modeling and Solution Algorithm for Optimization Integration of Express Terminal Nodes With a Joint Distribution Mode
}

\author{
Fanchao Meng, School of Computer Science and Technology, Harbin Institute of Technology at Weihai, China \\ Qingran Ji, School of Computer Science and Technology, Harbin Institute of Technology at Weihai, China \\ Hongzhen Zheng, School of Computer Science and Technology, Harbin Institute of Technology at Weihai, China \\ Huihui Wang, School of Computer Science and Technology, Harbin Institute of Technology at Weihai, China \\ Dianhui Chu, School of Computer Science and Technology, Harbin Institute of Technology at Weihai, China
}

\begin{abstract}
The rapid development of e-commerce has led to increased pressure on the express delivery industry to transport products to customers in a timely manner. The problem of how to deliver an increasing volume of express orders to customer clusters in a timely manner and at low cost with the joint distribution mode is becoming urgent. In this study, an express terminal node optimization and integration model is presented with an option to detach single customer clusters. In addition, the simulated annealing algorithm (SAA) based on neighborhood search that includes four rules is proposed to solve the problem. Contrast experiments are performed with SAA, the immune genetic algorithm (IGA), and the CPLEX solver. The experimental results indicate that IGA is less effective than SAA, and the running time of the IGA is longer. The CPLEX solver is less effective than the SAA, too. Additionally, the experimental results also show that every neighborhood rule proposed in this study plays a role in the optimization process.
\end{abstract}

\section{KEYWORDS}

Detachable Customer Business, Express Terminal Nodes Integration, Immune Genetic Algorithm, Minimum Cost, Neighborhood Search Rules, Simulated Annealing Algorithm

\section{INTRODUCTION}

With the rapid development of e-commerce, express delivery industry has been in a rapid development stage. Various multimedia and Internet technologies also have fueled strong cravings for express delivery industry information within our culture (Hazlewood \& Coyle, 2009). It has become one of the fastest growing industries in the world, especially in developing countries. According to statistics obtained from the China Post, the volume of express business reached 40.06 billion in 2017, which was an increase of $28 \%$ over the previous year (Duan, Song, Qu, Dong, \& Xu, 2019). It then reached 507.1 billion in 2018, which was an increase of $26.6 \%$ over the previous year (Duan, Song, Qu, Dong, $\& \mathrm{Xu}, 2019)$. In fact, the express delivery industry has maintained its rapid growth in many countries. The cost of global express deliveries, excluding pickup, linehaul, and sorting, is about EUR 70 billion, 
with China, Germany, and the United States accounting for more than $40 \%$ of the market (Duan et al., 2019). It is forecasted that delivery volumes in Germany and the U.S. could double over the next ten years (until 2025), reaching roughly 5 billion and 25 billion parcels per year, respectively (Joerss, Schröder, Neuhaus, Klink, \& Mann, 2016). The rapid development of e-commerce not only brings the express delivery industry unprecedented opportunities, but also many new problems and challenges. At present, the "last kilometer" distribution used by the express delivery industry poses a problem of how to deliver an increasing volume of goods to customer clusters in a timely manner and at a low cost (He, Wang, Lin, Zhou, \& Zhou, 2017). The implementation of an express terminal network with a joint distribution mode is an effective way to solve this problem. The joint distribution mode, which refers to the distribution behavior organized and implemented by more than one enterprise, originated in Japan in the 1960s. With this mode, less business from a single enterprise is centrally distributed. This mode improves the utilization rate of the vehicles used for deliveries, and breaks the restriction of distributions by individual enterprises (He et al., 2017; Sun, Karwan, Banu, \& Pinto, 2015; Wang et al., 2017). However, information sharing can cause privacy and security problems. Yamin et al. (2018) focus on this problem and introduce an approach based on the concept of cooperation between peers (P2P), facilitated by cache. This is not the main research content of this paper, but it should also be paid attention to.

Prior to implementing joint distribution, express delivery companies usually choose where to set express terminal nodes according to their own business. Therefore, it is ubiquitous that some express delivery companies repeatedly set nodes to compete with each other maliciously. This brings such problems as redundant number of nodes, low resource utilization rates for nodes, unreasonable node layouts, and high distribution cost (Ji, Yang, Zhang, \& Zhong, 2013). Owing to the previously mentioned problems, it is necessary to integrate optimized express terminal nodes from many express delivery companies in the same region to implement the joint distribution mode more efficiently. Sharing of logistics resources can be realized by integration. With the joint distribution mode, the optimization and integration of express terminal nodes is considered in order to reset the express terminal shared nodes by revoking and merging nodes. This process also redistributes business between the nodes and customer clusters when the supply of nodes set by different express delivery companies in the same region is greater than the demand of customer clusters. Hence, the optimized integration of express terminal nodes can improve the utilization rate of logistics resources and reduce the operation cost of express terminal nodes.

Figure 1 shows a schematic diagram that describes the optimization and integration of the express terminal nodes in a certain region. Three express delivery companies $(A, B$, and $C)$, eight nodes $\left(A_{1}\right.$, $\left.A_{2}, A_{3}, B_{1}, B_{2}, B_{3}, C_{1}, C_{2}\right)$, and seventeen customer clusters $(1,2, \ldots 17)$ were analyzed. Company $A$ had three nodes, company $B$ had three nodes, and company $C$ had two nodes. After optimal integration is achieved, four nodes were canceled, and the remaining were kept as shared nodes. The customer clusters were all covered by the shared nodes shown in the right hand image of Figure 1. Shared node $A_{3}$ covered customer clusters $9,11,12,14$, and 16 , shared node $B_{1}$ covered customer clusters $1,2,4$, 5,6 , and 9 , shared node $B_{2}$ covered customer clusters $2,3,6,7$, and 8 , and shared node $C_{2}$ covered customer clusters 10,13, 15, and 17. Customer clusters 2, 6, 9, 15, and 17 had their demand business distributed to different nodes called detachable customer clusters. The five detachable customer clusters are shown in the right hand image in Figure 1. For example, the business of customer cluster 2 was proportionally distributed to shared nodes $B_{1}$ and $B_{2}$. Analogously, the business of customer cluster 6 was proportionally assigned to shared nodes $B_{1}$ and $B_{2}$, the business of customer cluster 9 was proportionally assigned to shared nodes $B_{1}$ and $A_{3}$, the business of customer cluster 15 was proportionally assigned to shared nodes $A_{3}$ and $C_{2}$, and the business of customer cluster 17 was proportionally assigned to shared nodes $A_{3}$ and $C_{2}$. The problem to be solved in this study is how to choose which nodes should be retained as shared nodes. The issue of how to reasonably distribute the business of the customer clusters must also be considered. 


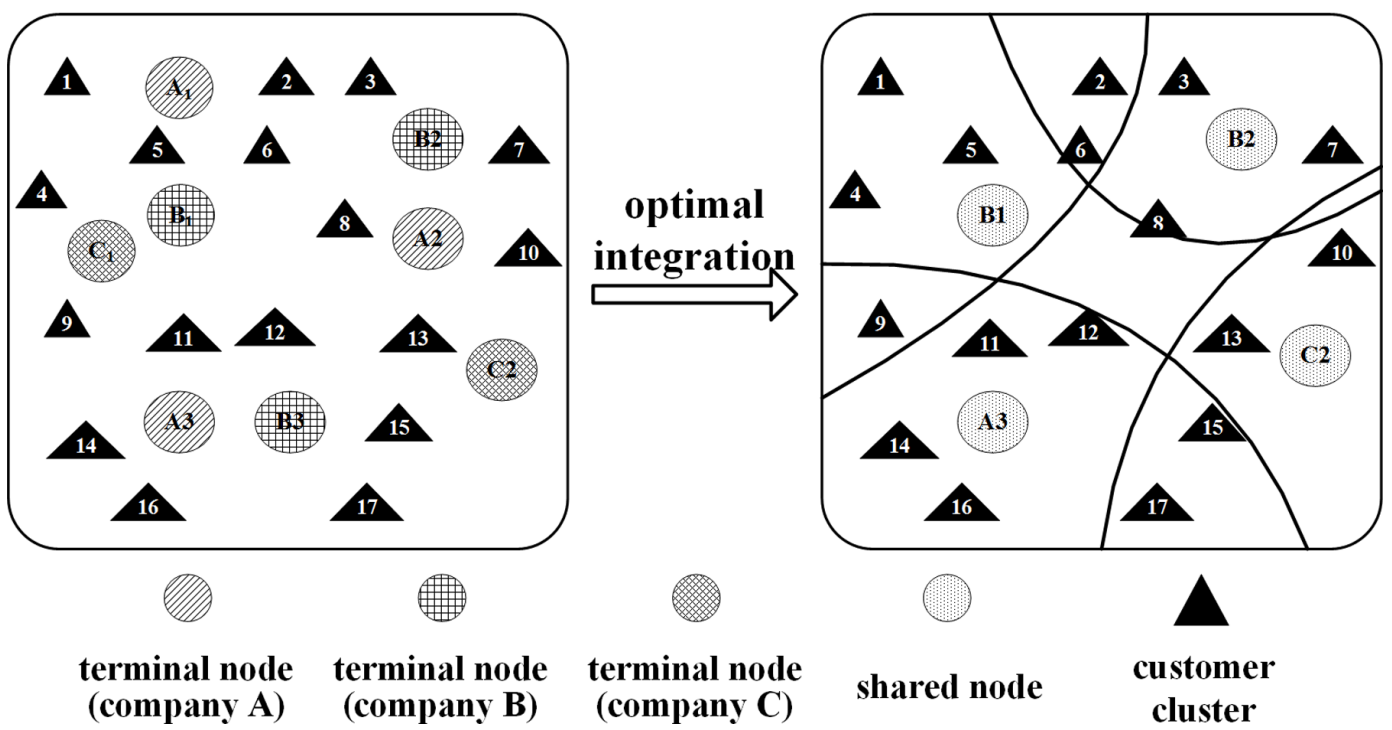

The express terminal nodes optimization integration problem (ETNOIP) is a NP-hard problem, with relatively little research available at present. One of the few related papers, by Wang et al. (2019), focused on the application of the immune genetic algorithm (IGA) to solve ETNOIP and established an express terminal network optimization and integration model to minimize the sum of the operation cost of the nodes and the transportation cost between the nodes and customer clusters. However, the model only considered how to distribute the overall business from one customer cluster to one shared node. This distribution method led to problems such as low resource utilization and unreasonable setting of nodes.

ETNOIP is similar to the logistics distribution center location problem (LDCLP), with both requiring a reasonable layout of nodes. LDCLP refers to the optimization problem of how to select suitable supply points as the distribution center points from the scope with a certain number of supply and demand points (Droździel, Wińska, Madleňák, \& Szumski, 2017). LDCLP can be transformed into a p-median problem, which is a typical NP-hard problem (Hakimi, 1964). Many approximation algorithms have been proposed to solve the LDCLP, including the heuristic algorithm (Guastaroba \& Speranza, 2014; Sun, Gao, \& Wu, 2008), clustering algorithm (Ji et al., 2013), genetic algorithm (Liu, Fan, Wang, \& Dong, 2009), ant colony algorithm (Ting \& Chen, 2013), and particle swarm algorithm (Hua, Hu, \& Yuan, 2016). However, the LDCLP requires predetermination of the number of distribution center nodes and does not consider the capacity of the supply points or situations where one supply point covers multiple demand points. Therefore, the existing models and algorithms typically used to solve the LDCLP are not suitable for the ETNOIP.

The ETNOIP model is similar to the model of a special location problem known as the capacitated fixed charge facility location problem (CFCFLP) (Nozick, 2001). Facility location is an important research area of many location problems. Many applications dependent on the location-based-services (LBS). For example, Yang et al. (2018) build an all-dimensional, stereoscopic and real-time integrated transportation network which needs to determine the location of transport facilities in advance. The optimization objective of the CFCFLP is to minimize the construction cost of all facilities and the transportation cost between the demand nodes and facilities. The CFCFLP can be expressed as a mixed integer programming model. Many efficient algorithms have been proposed to solve the problem, such as branch-and-bound (Klose \& Gortz, 2007), Lagrangian relaxation (Melkote \& Daskin, 2001), 
the heuristic algorithm (Wu, X.S. Zhang, \& J.L. Zhang, 2006), etc. The CFCFLP is also an NP-hard problem, and there is currently no effective polynomial algorithm to solve the problem. Researchers typically use approximate algorithms to obtain an approximate optimal solution. Jaramillo et al. (2002) used a genetic algorithm to solve the CFCFLP and compared the efficiency with other heuristic algorithms through open data sources. However, there are some defects in the encoding of this method that cannot express how to distribute business from one demand point to multiple facilities. Venables and Moscardini $(2006 ; 2008)$ solved the CFCFLP via some improved ant colony algorithms and used a series of benchmark data to verify the effect of the algorithms. These algorithms include ant colony optimization, the MAX-MIN Ant System, etc. For plant locations with capacity constraint issues, Kai et al. (2010) proposed a hybrid of the bender decomposition algorithm and genetic algorithm, with the hybrid algorithm producing better simulation results than the bender decomposition algorithm. Mahdian et al. (2006) proposed a 2-approximation algorithm to solve the Soft-CFCFLP, and Pál et al. (2001) put forward a local search algorithm with an approximate ratio of $9+\varepsilon$ whose neighborhood operations include ADD, CLOSE, and OPEN. On the basis of this study (Pál et al, 2001), Zhang et al. (2005) used MULTI-EXCHANGE instead of CLOSE / OPEN where the approximation ratio reached 5.83+E. Xu et al. (2019) applied a local search algorithm to solve the squared metric facility location problem with nonuniform capacities where the approximate ratio reached $13+\varepsilon$. However, the CFCFLP does not consider the scope of the nodes, which makes it difficult to directly apply existing algorithms to the ETNOIP.

Existing methods to solve ETNOIP also have some deficiencies. Research on these deficiencies is limited, and only considers methods to distribute the overall business from one customer cluster to one shared node, which leads to low resource utilization and unreasonable setting of nodes (Wang et al., 2019). The ETNOIP is similar to the LDCLP and CFCFLP. While, many efficient algorithms have been proposed to solve the LDCLP and CFCFLP, these algorithms are not suitable for solving the ETNOIP. In view of the shortcomings of the existing algorithms and models, this study proposes an express terminal nodes optimization and integration model with divisible business of customer clusters, and uses the simulated annealing algorithm (SAA) based on a neighborhood search that involves the four neighborhood rules to solve the problem. In order to verify the effectiveness of the algorithm proposed in this study, the IGA is implemented (Wang et al., 2019) and applied to the model. In addition, the problem is solved with the CPLEX, an IBM optimization solver. The results of the experiments show that the integration effect of the SAA is better than that of the IGA and CPLEX solver, and the runtime of the SAA is shorter than the IGA. It is also concluded that the IGA is not suitable for the model established in this study based on the experiment results. Thus, the contributions of this study are as follows:

- Contribution 1: An express terminal nodes optimization and integration model with divisible business of customer clusters is established to solve the ETNOIP that considers the capacity and scope of the express terminal nodes.

- Contribution 2: According to the model, four special neighborhood search rules are designed for neighborhood searches of the SAA, that enable the algorithm to search for optimal solutions to the maximum extent possible without destroying the original solution structure.

- Contribution 3: The experimental results of the SAA based on the neighborhood search are better than not only the IGA, but also the CPLEX solver. It is concluded that the IGA is not suitable for the model proposed in this study based on the experimental results.

The remainder of this paper is presented as follows: Section 2 describes the optimization and integration model of the express terminal nodes. Section 3 introduces the method utilized in this study. The SAA based on a neighborhood search was utilized to solve the ETNOIP. Section 4 introduces the method based on the IGA. Section 5 describes the comparison experiments and analyzes the 
experimental results. Section 6 summarizes the work from this study and introduces future research topics.

\section{EXPRESS TERMINAL NODES OPTIMIZATION AND INTEGRATION MODEL}

Under the joint distribution mode, cooperation and sharing between express delivery companies can lead to situations where more than one node covers the same area. This leads to the waste of node resources and an increase in the express transportation cost such that the supply of nodes exceeds the demand of customer clusters. In order to solve this problem, the express terminal nodes must be integrated reasonably, and redundant nodes must be canceled. The problem of node integration transforms into a task distribution problem that reasonably distributes the business of customer clusters to nodes and cancels the express terminal nodes that business is not distributed to, based on coordination of supply and demand. In fact, the business of one customer cluster can be distributed to more than one node. One node can cover more than one customer cluster and one customer cluster can be covered by more than one node, therefore, the distribution relationship between customer clusters and nodes is many to many in the model.

In this study, authors used natural numbers to code the nodes and customer clusters. Let $n$ and $m$ be the number of customer clusters and nodes, respectively, $I=\left\{c c_{1}, c c_{2}, \ldots, c c_{i}, \ldots c c_{m}\right\}$ represent the set of customer clusters where $c c_{i}$ represents the $i_{t h}$ customer cluster in $I$, and $J=\left\{n d_{1}, n d_{2}, \ldots, n d_{j}, \ldots\right.$ $n d_{n}$ \} represent the set of nodes where $n d_{j}$ represents the $j_{t h}$ node in $J$. For every $n d_{j}$, let $c_{j}$ represent the operating cost of $n d_{j}, d_{j}$ represent the business coverage of $n d_{j}$, and $f_{j}$ represent the maximum business capacity of $n d_{j}$. For every $c c_{i}$, let $g_{i}$ represent the business demand of $c c_{i}$. Let $d_{i j}$ represent the distance between $c c_{i}$ and $n d_{j}$, and $u_{i j}$ represent the unit transportation cost between $c c_{i}$ and $n d_{j}$ which is the transportation cost per unit business and per unit distance, respectively.

Table 1 lists the main variables' descriptions used in this study. Let $x_{i j}$ and $y_{j}$ be the decision variables for the problem. The variable $x_{i j} \in\left\{0,1, \ldots, \min \left\{f_{j}, g_{i}\right\}\right\}$ is a nonnegative integer decision variable that represents the business distributed from $c c_{i}$ to $n d_{j}$. If $x_{i j}=0, n d_{j}$ does not cover $c c_{i}$ or any business of $c c_{i}$ is not assigned to $n d_{j}$. If $x_{i j} \neq 0, n d_{j}$ covers $c c_{i}$ and the business of $c c_{i}$ distributed to $n d_{j}$ is $x_{i j}$. The variable $y_{j} \in\{0,1\}$ is a binary decision variable. If $y_{j}=1, n d_{j}$ is kept; otherwise, $n d_{j}$ is canceled. The value of $y_{j}$ is related to $x_{i j}$, and is given by:

$y_{j}=\left\{\begin{array}{rc}0, & \sum_{i=1}^{n} x_{i j}=0 \\ 1, & \text { else }\end{array}\right.$

Under the mode of joint distribution, the express terminal nodes optimization and integration model can be formally defined as follows:

$$
\min \sum_{j=1}^{m} c_{j} y_{j}+\sum_{i=1}^{n} \sum_{j=1}^{m} d_{i j} u_{i j} y_{j} x_{i j}
$$

s.t.

$$
\left(d_{j}-d_{i j}\right) x_{i j} \geq 0 \quad i=1,2, \ldots, m ; j=1,2, \ldots, n
$$


$\sum_{i=1}^{n} x_{i j} \leq f_{j} y_{j} \quad j=1,2, \ldots, n$

$\sum_{j=1}^{m} x_{i j}=g_{i} \quad i=1,2, \ldots, m$

$y_{j} \in\{0,1\} \quad j=1,2, \ldots, n$

$x_{i j} \in\left\{0,1, \ldots, \min \left\{f_{j}, g_{i}\right\}\right\} \quad i=1,2, \ldots, m ; j=1,2, \ldots, n$

where Eq. (2) is the optimization target to obtain the lowest total cost, which includes the operational cost of the nodes and the transport cost between the nodes and customer clusters. Eq. (3) is the distance constraint whereby every node provides business only for customer clusters that are located in its coverage. Eq. (4) shows that the business distributed to one node cannot exceed its business capacity, and Eq. (5) shows that all of the business from every customer cluster must be distributed to corresponding nodes. Eqs. (6) and (7) are the constraints for the decision variables.

\section{THE SAA BASED ON NEIGHBORHOOD SEARCH}

The SAA was first proposed by N. Metropolis et al. in 1953. In 1983, S. Kirkpatrick et al. successfully introduced it into the field of combinatorial optimization. They considered that there was a similarity

Table 1. Primary variable descriptions

\begin{tabular}{|c|c|c|}
\hline Variable type & Symbol & Definition \\
\hline \multirow{12}{*}{ Ordinary variables } & $n$ & The number of nodes \\
\hline & $m$ & The number of customer clusters \\
\hline & $J$ & The set of nodes \\
\hline & $I$ & The set of customer clusters \\
\hline & $j$ & The serial number of nodes \\
\hline & $i$ & The serial number of customer clusters \\
\hline & $c_{j}$ & The operation cost of $n d_{j}$ \\
\hline & $d_{j}$ & The coverage of $n d_{j}$ \\
\hline & $f_{j}$ & The maximum business capacity of $n d_{j}$ \\
\hline & $g_{i}$ & The business demand of $c c_{i}$ \\
\hline & $d_{i j}$ & The distance between $c c_{i}$ and $n d_{j}$ \\
\hline & $u_{i j}$ & The unit transportation cost between $c c_{i}$ and $n d e_{j}$ \\
\hline \multirow{2}{*}{ Decision variables } & $x_{i j}$ & The business distributed from $c c_{i}$ to $n d_{j}$ \\
\hline & $y_{j}$ & The operation status of $n d_{j}$ \\
\hline
\end{tabular}


between the annealing process of solid matter in physics and the general combinatorial optimization problem. The SAA can effectively avoid the objective function falling into a local minimum with a probability jump, which is time-varying and eventually tends to zero with the search process. In solving the ETNOIP, the SAA can jump out of the local optimal solution with probability, before it finally tends to the global optimal solution. There are some acceptance criteria to judge whether the new solution is acceptable. The most frequently used criterion is the Metropolis rule to generate a random number, $\varepsilon \in[0,1)$. If the acceptance probability, $p$, is greater than $\varepsilon$, the new state is accepted as the current state. Otherwise, the original state is retained as the current state. This is expressed by:

$$
P=\left\{\begin{array}{cc}
1 & E\left(X_{\text {new }}\right) \leq E\left(X_{\text {old }}\right) \\
\exp \left(\frac{E\left(X_{\text {new }}\right)-E\left(X_{\text {old }}\right)}{T}\right) & E\left(X_{\text {new }}\right)>E\left(X_{\text {old }}\right)
\end{array}\right\}
$$

where $X_{\text {new }}$ represents the new state, $E\left(X_{\text {new }}\right)$ represents the energy of the new state, $X_{\text {old }}$ represents the original state, and $E\left(X_{\text {old }}\right)$ represents the energy of the original state.

According to the model established in Section 2, the SAA is designed and implemented based on a neighborhood search to solve the problem. Additionally, the initialization rule and four neighborhood search rules are defined.

\section{Encoding of The Solution}

The business of one customer cluster can be assigned to more than one node. One node can cover more than one customer cluster and one customer cluster can be covered by more than one node, therefore, the distribution relationship between the customer clusters and nodes is many to many in the model. A two-dimensional matrix of $m^{*} n$ and one-dimensional array with a length of $n$ are defined as the encoding of the solution. The two-dimensional matrix represents the decision variable, $x$, and the one-dimensional array represents the decision variable, $y$. The variable $m$ represents the number of customer clusters, while $n$ represents the number of nodes. In the two-dimensional matrix, the value of the $i_{t h}$ row and $j_{t h}$ column, $x_{i j}$, is the business distributed from $c c_{i}$ to $n d_{j}$. In the one-dimensional array, the $j_{t h}$ array element, $y_{j}$, represents the operation status of $n d_{i j}$.

Figure 2 shows an example of encoding where $m=7$ and $n=5$. This example is used to explain the initialization rule and the four neighborhood rules. In the left side of Figure 2, the seven triangles represent seven customer clusters and the numbers on triangles represent the business demand of customer clusters. The five circles represent five nodes and the numbers on circles represent the maximum business capacity of nodes. The right side of Figure 2 represents the decision variables and some other variables. For example, the number in the right side of Figure 2, the first row and first column of $x$ is equal to 161 , which represents the business distributed from $c c_{1}$ to $n d_{1}$. The value of the first element in $y$ is equal to 1 , which indicates that $n d_{1}$ is retained. The value of the fifth element in $y$ is equal to 0 , which indicates that node $n d_{5}$ is canceled. In the right side of Figure 2 , the second column from the right represents the business of each customer cluster, the first column from the right represents the business distribution rate of each customer cluster, the third row from the bottom represents the business occupied by each node, and the second row from the bottom represents the business occupancy rate of each node.

\section{The Initialization Rule of The Solution}

Before using the SAA to solve the problem, an initialization rule of the solution is defined in order to produce a better initial solution that will ultimately improve the probability of finding the global optimal solution. The initialization rule of the solution is given by algorithm 1 . The principle of algorithm 1 is similar to Greedy Algorithm. When distributing some business from one customer 
Figure 2. Schematic diagram of the solution encoding

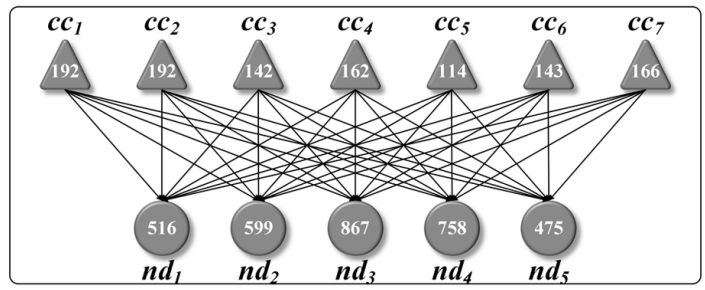

$c c_{i}, \mathbf{i} \in \mathbf{I}, \mathbf{0} \leqslant \mathbf{i} \leqslant \mathbf{m}, \mathbf{g}_{\mathbf{i}}$ is the business of $c c_{i}$

$n d_{j}, j \in J, 0 \leqslant j \leqslant n, f_{j}$ is the maximum capacity of $n d_{j}$
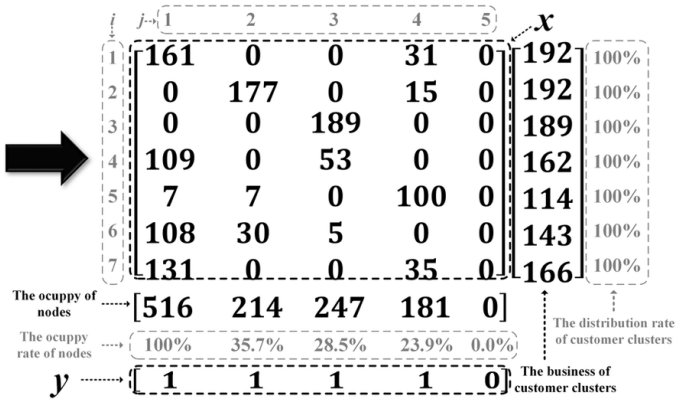

Table 2. The major variables and functions of algorithm 1-5

\begin{tabular}{|c|c|c|c|c|}
\hline variables & \multicolumn{2}{|l|}{ type } & \multicolumn{2}{|c|}{ representation } \\
\hline$x[1 \ldots m][1 \ldots n]$ & \multicolumn{2}{|c|}{ two-dimensional matrix } & \multicolumn{2}{|c|}{ The solution } \\
\hline nd_Occupy $[1 \ldots n]$ & \multicolumn{2}{|c|}{ one-dimensional array } & \multicolumn{2}{|c|}{ The business occupied by each node } \\
\hline$c c_{-}$Rest $[1 \ldots m]$ & \multicolumn{2}{|c|}{ one-dimensional arrays } & \multicolumn{2}{|c|}{ The business to be distributed to each customer cluster } \\
\hline$d \_\operatorname{Cost}[1 \ldots m][1 \ldots n]$ & \multicolumn{2}{|c|}{ two-dimensional matrix } & \multicolumn{2}{|c|}{ The distribution cost between each node and customer } \\
\hline cancelList $[1 \ldots l]$ & \multicolumn{2}{|l|}{ one-dimensional array } & \multicolumn{2}{|c|}{ The randomly generated cancellation sequence } \\
\hline$f[1 \ldots n]$ & \multicolumn{2}{|c|}{ one-dimensional arrays } & \multicolumn{2}{|c|}{ The maximum business capacity of each node } \\
\hline$d 1[1 \ldots n]$ & \multicolumn{2}{|c|}{ one-dimensional arrays } & \multicolumn{2}{|c|}{ The coverage of each node } \\
\hline$d 2[1 \ldots m][1 \ldots n]$ & \multicolumn{2}{|c|}{ two-dimensional matrix } & \multicolumn{2}{|c|}{ The distance between each node and customer } \\
\hline functions name & parameter & \multicolumn{2}{|c|}{ return } & function \\
\hline calculateCost & nd_Occupy, cc_Rest & \multicolumn{2}{|c|}{$d \_C o s t$} & calculate the $d \_$Cost by formula (11) \\
\hline calculateNdOccupy & $x$ & \multicolumn{2}{|c|}{ nd_Occupy } & calculate the $n d \_O c c u p y$ by formula (9) \\
\hline calculateCcRest & $x$ & \multicolumn{2}{|c|}{$c c \_$Rest } & calculate the $c c \_$Rest by formula (10) \\
\hline $\min C o s t i$ & $d \_$Cost & \multicolumn{2}{|c|}{$d_{-}$Costi } & get the subscripts $i$ of the minimum in $d_{-}$Cost; \\
\hline $\min C o s t j$ & $d \_$Cost & \multicolumn{2}{|c|}{$d \_C o s t j$} & get the subscripts $i$ of the minimum in $d_{-}$Cost; \\
\hline roulette & $x$ & \multicolumn{2}{|c|}{ node_num } & select node by roulette selection method \\
\hline selectLowNode & $x$ & \multicolumn{2}{|c|}{ node_num } & select node with low utilization \\
\hline
\end{tabular}

to one node, the distribution cost is lower, the more the distribution scheme will be selected. The declaration of variables and functions is shown in Table 2.

The nodeOccupy[1...n] and customerRest [1...m] can be calculated by formula (9) and (10) .

$n d_{-}$Occupy $_{j}=\sum_{i=1}^{m} x_{i j}, j=1,2, \ldots, n$ 


$$
c c_{-} \text {Rest }_{i}=g_{j}-\sum_{j=1}^{n} x_{i j}, i=1,2, \ldots, m
$$

The $d_{-}$Cost $[1 \ldots m][1 \ldots n]$ can be calculated by formula (11). If $c c_{i}$ is not in the scope of $n d_{j}$, the value $d_{-}$Cost $_{i j}$ is $\infty$. When distributing the business of $c c_{i}$ to $n d_{j}$, if the business occupied by $n d_{j}$ is not zero, the value of $d_{-}$Cost $_{i j}$ is equal to the product of the business to be distributed from $c c_{i}$ and the unit transportation cost. If the business occupied by $n d_{j}$ is zero, the value of $d_{-}$Cost $_{i j}$ must include the operational cost of $n d_{j}$. When distributing, give priority to the distribution scheme with lower value of $d_{-}$Cost $_{i j}$.

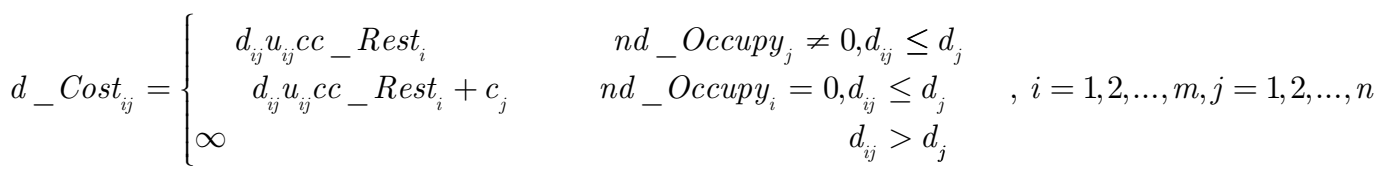

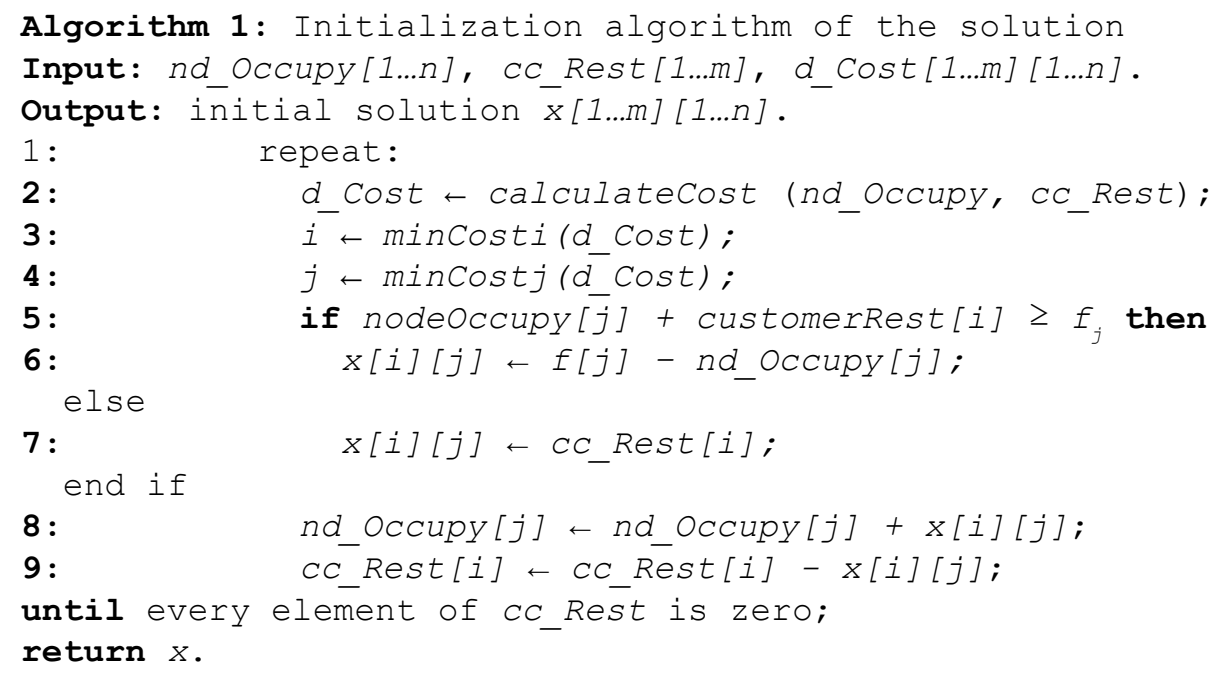

\section{The Neighborhood Operations}

Four neighborhood search rules are defined for this study.

Rule 1: cancel and redistribute business of some customer clusters operation

This operation is executed with a probability greater than $p_{a}$. A one-dimensional array called cancelList is defined that saves some randomly generated numbers $\left\{c l_{1,} c l_{2, \ldots . .,} c l_{k,} \ldots \ldots, c l_{l}\right\}$. The length of cancelList is $l$, which is a random number less than $m$ (i.e., the number of customer clusters). Then, the business distribution schemes of the customer clusters whose serial numbers belong to cancelList are canceled, and their business is redistributed based on the principle of the lowest distribution cost like Algorithm 1.

The operation diagram is shown in Figure 3. Suppose that the cancelList is $\{1,6,7\}$. The business distribution schemes of $c c_{1}, c c_{6}$, and $c c_{7}$ are canceled and redistributed. The declaration of variables and functions is shown in Table 2. The algorithm is given by:

Algorithm 2: Cancellation and redistribution of the business of some customer clusters algorithm 
Figure 3. Schematic diagram of neighborhood rule 1
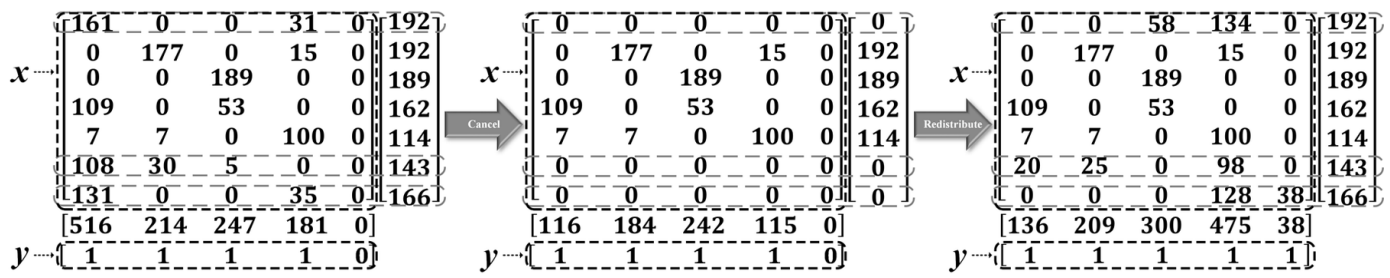

Input: current solution $x[1 \ldots m][1 \ldots n]$, cancelList[1...1].

Output: new solution $x[1 \ldots m][1 \ldots n]$ after rule 1 .

for $i:=1$ to 1

1:

end for

2: $\quad$ nd_occupy $\leftarrow$ calculateNdOccupy $(x)$;

3: $\quad$ cC_Rest $\leftarrow$ calculatecc_Rest (x);

Call Algorithm 1

return $x$.

Rule 2: exchange business of some nodes operation

This operation is executed with a probability greater than $p_{b}$. Two numbers, node_ran 1 and node_ran 2 , are randomly generated and then the business distributed to $n d_{\text {node_ran1 }}$ and $n d_{\text {node_ran } 2}$ is exchanged.

The operation diagram is shown in Figure 4. Suppose that the numbers randomly generated are 2 and 3 . The business distributed to $n d_{2}$ and $n d_{3}$ is exchanged. The declaration of variables and functions is shown in Table 2. The algorithm is given by:

Algorithm 3: Exchange business of some nodes algorithm

Input: current solution $x[1 \ldots m][1 \ldots n]$.

Output: new solution $x[1 \ldots m][1 \ldots n]$ after rule 2 .

for $i:=1$ to $m$

1: if $d 2$ [i] [node_ran1] $\leq d 2$ [node_ran1] \&\&d2[i] [node_ ran2] $\leq d 2$ [node_ran2] then

$x[i][$ node_ran1] $\langle->x[i][$ node_ran2];

end if

Figure 4. Schematic diagram of neighborhood rule 2

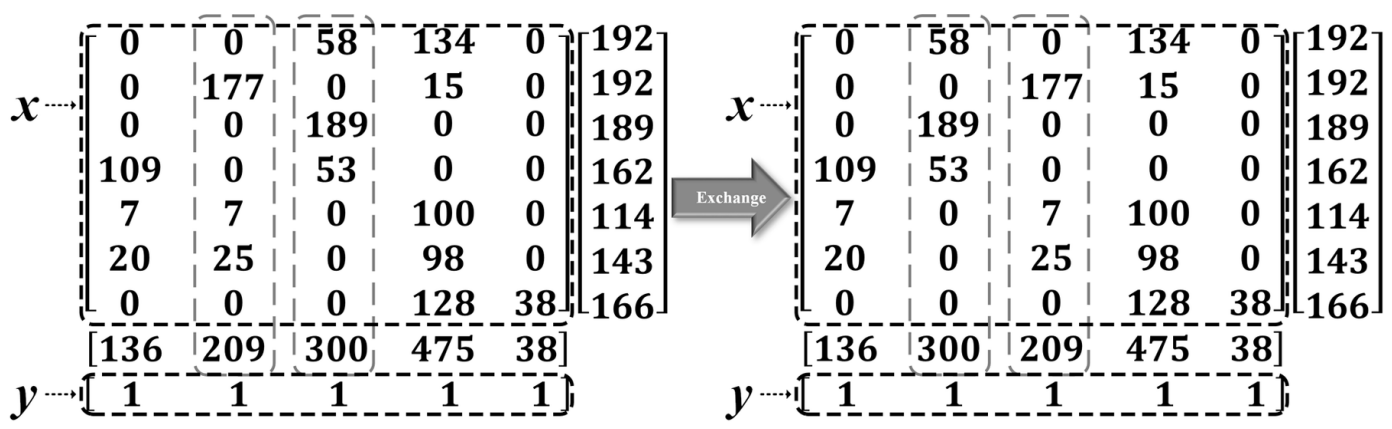


Figure 5. Schematic diagram of neighborhood rule 3

\begin{tabular}{|c|c|c|c|c|c|c|c|c|c|c|c|c|c|c|c|c|c|}
\hline 0 & $5 \overline{8}-$ & 0 & 134 & $\left.\overline{0}^{0}\right]\left[\begin{array}{l}192 \\
\end{array}\right.$ & i & 0 & $5 \overline{8}$ & 134 & 0 & 192 & {$[\bar{c}$} & $\overline{\mathbf{0}}$ & 58 & 0 & 134 & 0 & 57 \\
\hline $\mathbf{0}$ & $\mathbf{0}$ & 177 & 15 & 192 & 0 & 177 & $\mathbf{0}$ & 15 & $\mathbf{0}$ & 192 & & $\mathbf{0}$ & $\mathbf{0}$ & 177 & 15 & $\mathbf{0}$ & 192 \\
\hline $\mathbf{0}$ & 189 & 0 & 0 & 189 & 0 & 0 & 189 & 0 & $\mathbf{0}$ & 189 & & 0 & 189 & 0 & $\mathbf{0}$ & $\underline{0}$ & 189 \\
\hline 109 & 53 & $\mathbf{0}$ & $\mathbf{0}$ & 162 & 0 & $\mathbf{0}$ & 53 & 0 & 0 & 53 & c & 0 & 53 & 0 & 0 & 109) & 162 \\
\hline 7 & $\mathbf{0}$ & 7 & 100 & 114 & $\mid 0$ & 7 & $\mathbf{0}$ & 100 & $\mathbf{0}$ & 107 & 7 & $\mathbf{0}$ & $\mathbf{0}$ & (14) & 100 & 0 & 114 \\
\hline 20 & 0 & 25 & 98 & 143 & 0 & 25 & $\mathbf{0}$ & 98 & 0 & 123 & & $\mathbf{0}$ & $\mathbf{0}$ & (45) & 98 & $\mathbf{0}$ & 143 \\
\hline 0 & 0 & 0 & 128 & 38] & il & 0 & 0 & 128 & 38 & 166 & & 0 & 0 & $\overline{0}$ & 128 & 38 & "L166 \\
\hline 136 & 300 & 209 & 475 & 38] & 0 & 209 & 300 & 475 & 38] & & & {$[0$} & 300 & 236 & 475 & $147]$ & \\
\hline & 1. & $\mathbf{1}$ & 1 & 1] & & 1 & 1 & 1 & 1 & & & & 1 & 1 & -1 & 1 & \\
\hline
\end{tabular}

end for

return $x$.

\section{Rule 3: Move Out The Business of Some Nodes With High Distribution Costs Operation}

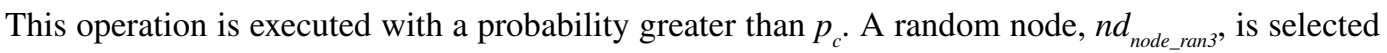
and its business is moved out and redistributed based on the principle of the lowest distribution cost like Algorithm 1. In order to let the new solution after the neighborhood operation that is closer to the optimal solution, neighborhood rule 3 refers to the selection operation in the genetic algorithm and uses the roulette selection method to randomly select a node and carry out neighborhood rule 3 .

The roulette selection method is a random selection algorithm based on probability and is also known as the proportional selection method. The basic idea is that the probability of each individual being selected is directly proportional to its fitness. In neighborhood rule 3 , the higher the total cost of one node is, the greater its fitness. The fitness calculation formula for $n d_{j}$ is given by:

$$
\text { fitness }_{j}=\left\{\begin{array}{cr}
\sum_{i=1}^{m} d_{i j} u_{i j} x_{i j}+c_{j} & \text { nodeOccupy }_{j} \neq 0 \\
0 & \text { nodeOcсupy }_{j}=0
\end{array}\right\}
$$

Let the selection probability of $n d_{j}$ for a single node be $P_{j}$, and the cumulative probability be $S_{j}$. The calculation formula is given by:

$$
\begin{aligned}
& P_{j}=\frac{\text { fitness }_{j}}{\sum_{j=1}^{n} \text { fitness }_{j}} \\
& S_{j}=\sum_{k=1}^{j} P_{k}
\end{aligned}
$$

A random number, $q$, is generated at $[0,1]$. If $q$ falls into $\left[0, S_{1}\right], n d_{1}$ is selected. If $q$ falls into $\left(S_{j-1}\right.$, $\left.S_{j}\right], n d_{j}$ is selected. Let roulette $(x)$ represent the function of randomly selecting nodes by the roulette selection method, and return the number of the selected node as node_num.

The operation diagram is shown in Figure 5. Suppose that the selected node is $n d$, using the roulette selection method. The business distributed to $n d_{1}$ from $c c_{4}, c c_{5}$, and $c c_{6}$ is moved to $n d_{3}$ and $n d_{5}$. The declaration of variables and functions is shown in Table 2 . The algorithm is given by:

Algorithm 4: Move out the business of some nodes with high

Input: current solution $x[1 \ldots m][1 \ldots n]$. 
Output: new solution $x[1 \ldots m][1 \ldots n]$ after rule 3 .

1: $\quad$ node_num $\leftarrow$ roulette $(x)$;

cancel the business distributed to nd node num from every customer;

2: $\quad$ nd_Occupy $\leftarrow$ calculateNdOccupy $(x)$;

3: $\quad$ cC Rest $\leftarrow$ calculatecc Rest (x) ;

Call Algorithm 1

return $x$.

Rule 4: move out the business of some nodes with low utilization rates operation

This rule moves out the business of some nodes with low utilization and redistributes business based on the principle of the lowest distribution cost like Algorithm 1. The difference between rule 4 and rule 3 is that rule 3 moves out the business of nodes with a high cost, while rule 4 moves out the business of nodes with low utilization rates. A threshold of $p_{d}$ is set, and a node with a utilization rate of less than $p_{d}$ is randomly selected to have its business moved out. Set nd_OccupyRate as the utilization rate of $n d_{j}$. The definition is given by:

$n d_{-}$OccupyRate $_{j}=\frac{n d_{-} \text {Occupy }_{j}}{f_{j}}$

The operation diagram is shown in Figure 6. Suppose that the selected node is $n d_{5}$. The business distributed to $n d_{5}$ from $c c_{4}$ and $c c_{7}$ is moved to $n d_{3}$ and $n d_{4}$. The declaration of variables and functions is shown in Table 2. The algorithm is given by:

Algorithm 5: Move out the business of some nodes with low utilization rates algorithm

Input: current solution $x[1 \ldots m][1 \ldots n]$.

Output: new solution $x[1 \ldots m][1 \ldots n]$ after rule 4.

1:

node_num $\leftarrow$ selectLowNode $(x)$

Figure 6. Schematic diagram of neighborhood rule 4

$x=\left[\begin{array}{cccccc}x \\ 0\end{array}\right.$

cancel the business distributed to nd node_num from every customer;

2: $\quad$ nd Occupy $\leftarrow$ calculateNdOccupy $(x)$;

3: $\quad$ cC_Rest $\leftarrow$ calculatecC_Rest (x);

Call Algorithm 1

return $x$. 


\section{The SAA Based on A Neighborhood Search}

The flowchart of the SAA based on a neighborhood search is shown on the left side of Figure 7. The algorithm steps are as follows:

Input: Information of the node set and customer set. Output: The distribution scheme, $x$, between the customer clusters and nodes, and the operation status, $y$, of the nodes. Initialize the algorithm parameters, including initial temperature, $t_{0}$, termination temperature, $t_{\text {end }}$ cooling coefficient, $a$, iteration number, $N$, cancel parameter, $p_{a}$, exchange probability, $p_{b}$, moving out probability, $p_{c}$, and utilization rate threshold, $p_{d}$.

In accordance with algorithm 1 , generate the initial solution $\left\{x_{0}, y_{0}\right\}$, and calculate the objective function, which is the total cost $f\left(x_{0}, y_{0}\right)$. Set $\left\{x_{0}, y_{0}\right\}$ as the current solution $\{x, y\}$ and the global best solution best_XY. The best objective function best solution is $f(x, y)$.

Set the current temperature $T=t_{0}$ and the current iteration number $n=0$.

In accordance with algorithms 2 to 5, execute the four neighborhood operations to get the new solution $\left\{x^{\prime}, y^{\prime}\right\}$. Calculate the objective function of $\left\{x^{\prime}, y^{\prime}\right\}$, which is the total cost $f\left(x^{\prime}, y^{\prime}\right)$. If $f\left(x^{\prime}, y^{\prime}\right)$ is less than $f(x, y)$, set $\left\{x^{\prime}, y^{\prime}\right\}$ as the current solution $\{x, y\}$ and the best objective function as best_solution $f\left(x^{\prime}, y^{\prime}\right)$. If not, according to Metropolis rule, determine whether to accept the new solution. If accepted, set $\left\{x^{\prime}, y^{\prime}\right\}$ as current solution $\{x, y\}$.

Set $n=n+1$ and then determine whether $n$ has reached the iteration number $N$. If $n$ has reached $N$, jump to step7. If not, jump to

\section{Step4}

Set $T=T^{*} a$ and then determine whether $T$ has reached the termination temperature, $t_{\text {end }}$. If $T$ has reached $t_{\text {end }}$ jump to step8. If not, set $n=0$ and jump to step4.

Output the best solution $\{x, y\}$ and the best function $f(x, y)$. End.

The time complexity of the above algorithm is $O\left(N \times \log _{\alpha} \frac{t_{\text {end }}}{t_{0}} \times m \times n\right)$ where $t_{0}$ is the initial temperature, $t_{\text {end }}$ is the termination temperature, $a$ is the cooling coefficient, $N$ is the iteration number, $m$ is the number of customer clusters, and $n$ is the number of nodes. $N, t_{\text {end }}, t_{0}$, and $\alpha$ are fixed, while $m$ and $n$ change according to the input of the algorithm.

\section{THE IMMUNE GENETIC ALGORITHM}

In the existing research (Wang et al., 2019), the immune genetic algorithm (IGA) is used to solve the problem. In contrast to the model proposed in this study, Wang et al. only consider how to distribute all business from one customer cluster to one node. In this study, while implementing the IGA from that study, the IGA is used to solve the model established in Section 2. In order to distinguish between the two algorithms, the former is called the undetachable immune genetic algorithm (UIGA), and the latter is called the detachable immune genetic algorithm (DIGA). 

follows:

The flowchart of the IGA is shown on the right side of Figure 7, and the algorithm steps are as

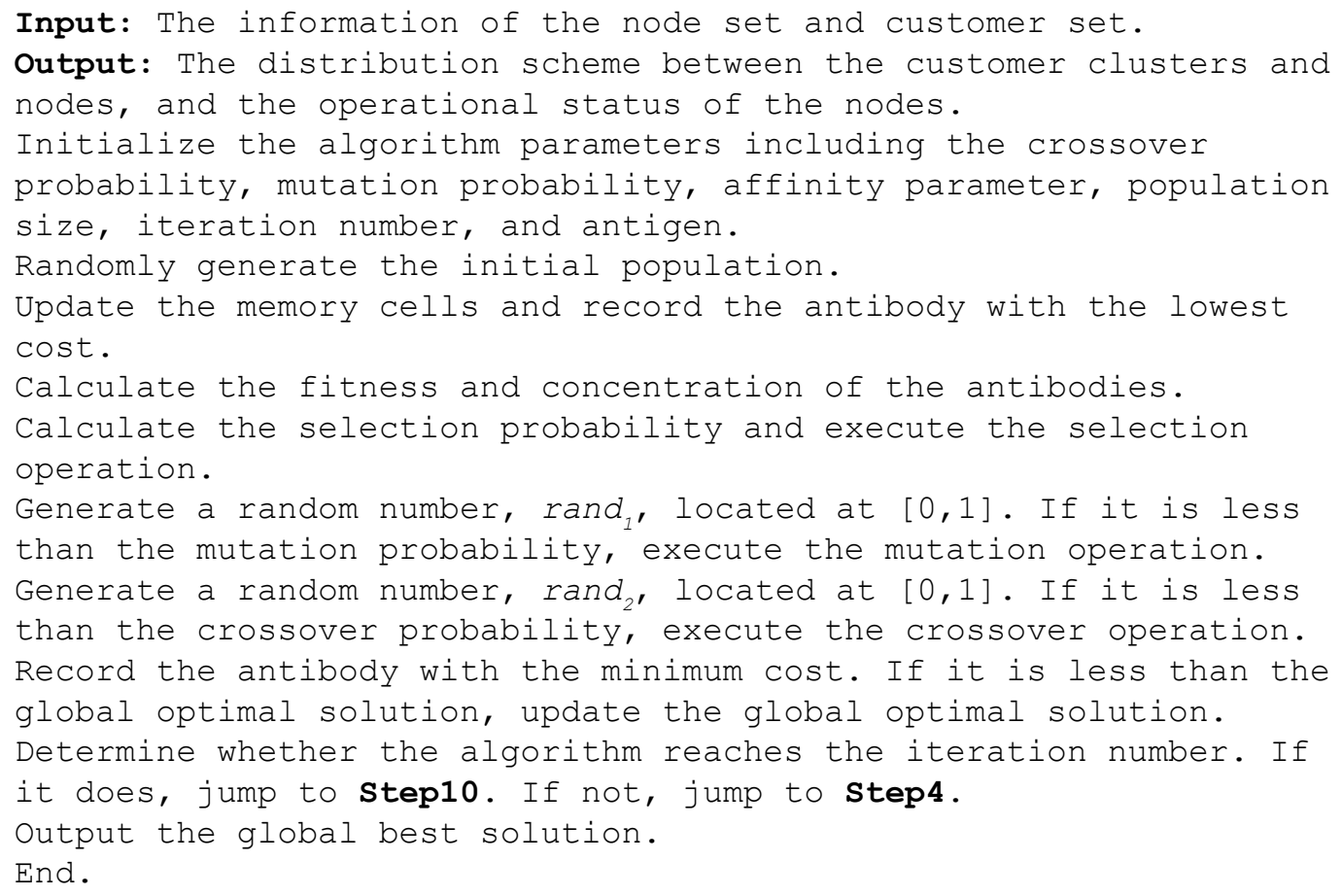

\section{Encoding of The Antibody}

Every antibody represents a distribution scheme. The encoding of an antibody with the traditional solution method is a one-dimensional array as shown in Figure 8. The antibody size is the number of customer clusters, and the value is the serial number of nodes, which means that all of the business from one customer cluster is distributed to one node. The encoding of the antibody in the case of detachable business is shown in Figure 2. This is the same as the encoding used in this study, which is a two-dimensional matrix.

\section{The mutation Operation}

In the traditional solution method, a number, $i$, less than the number of customer clusters and a number, $j$, less than the number of nodes is randomly generated, and all the business of $c c_{i}$ is redistributed to $n d_{j}$. In the case of detachable business, the mutation operation redistributes the business of $c c_{i}$ originally distributed to $n d_{j}$ to other nodes.

\section{The Crossover Operation}

In the traditional solution method, two numbers, $i_{1}$ and $i_{2}$, which are less than the number of customer clusters are randomly generated. Number $i_{1}$ is the starting point and number $i_{2}$ is the ending point of the crossover operation. In the case of detachable business, the crossover operation randomly generates two numbers, $j_{l}$ and $j_{2}$, which are less than the number of nodes, and exchanges the business distributed to every node that belongs to $\left[n d_{j 1}, n d_{j 2}\right]$. 
Figure 7. Flowchart of the SAA and IGA
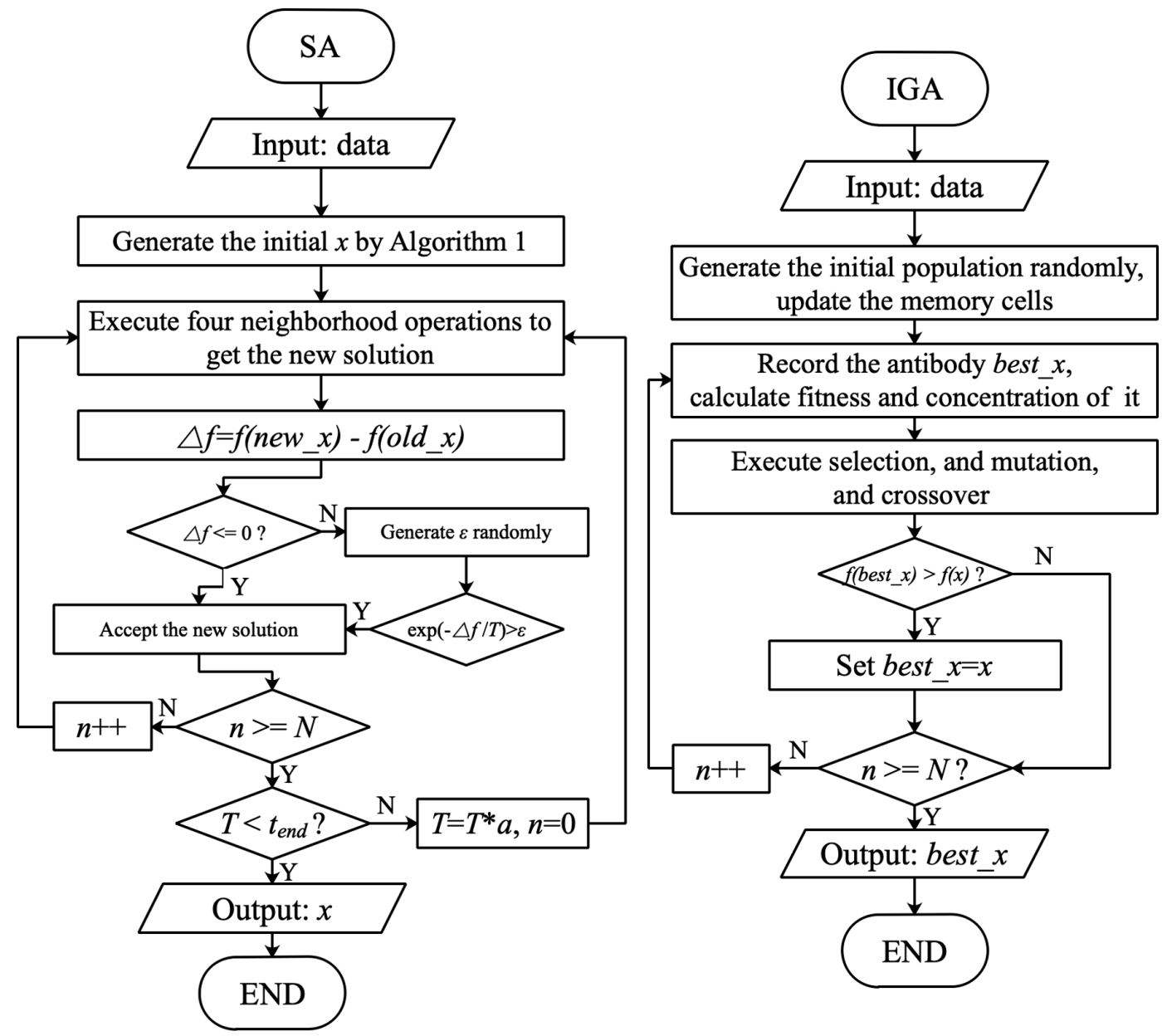

\section{EXPERIMENTAL SETUP AND RESULTS}

Figure 8. Encoding of the antibody using the traditional solution method

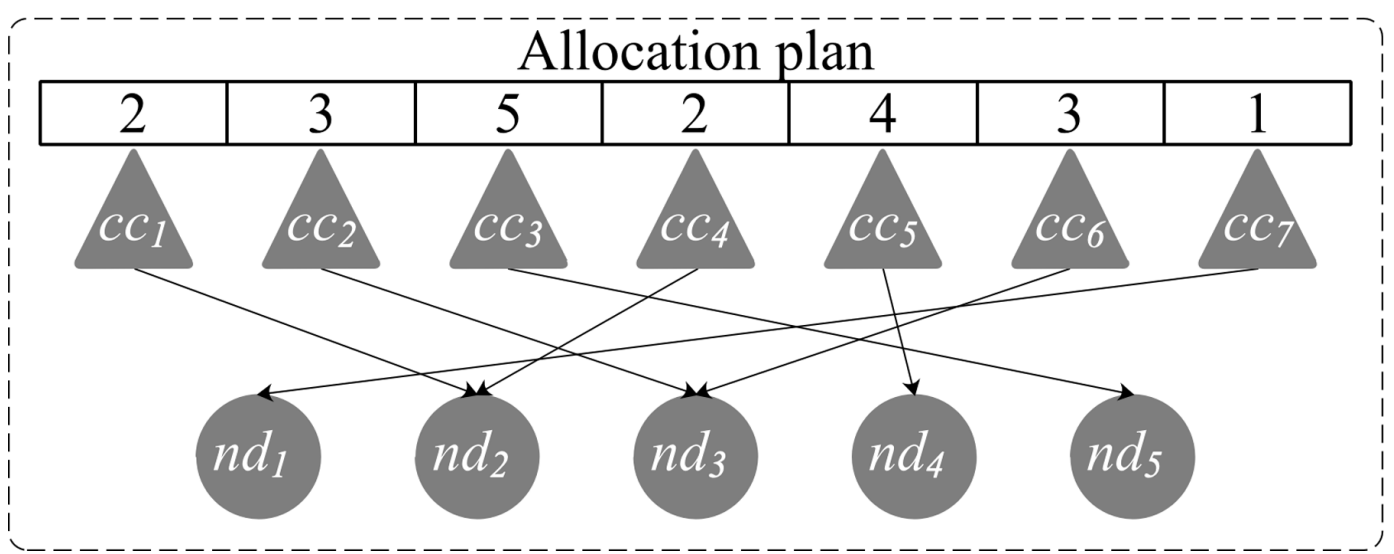


The experiments in this study were implemented on a Lenovo laptop with an Intel (R) core (TM) i55257u CPU @ 2.70 GHZ, with 4.0 GB of memory and a 64 bit operating system. In the experiments, the SAA based on a neighborhood search was used to solve the problem. At the same time, the UIGA and DIGA were realized. Finally, the results were compared with the commercial solver CPLEX12.7.1. All algorithms were developed in Java.

In order to further verify the effectiveness of the SAA based on a neighborhood search in solving the ETNOIP, it was compared with other algorithms and itself in a specially designed experiment. Four neighborhood operations were proposed in this study. The experiment was designed to remove one neighborhood operation and compare the result using only three neighborhood operations with the original result to analyze which neighborhood operation played a main role in the optimization of the problem.

\section{Experimental Configuration Parameters}

The experimental data was generated by simulation via the experimental configuration parameters, which were set by referring to the express logistics data of several cities in China. The test cases were determined by eight experimental configuration parameters, which are listed in Table 3.

The scale of the experiments could be changed by the number of nodes, $n$, the number of customer clusters, $m$, and the relationship type, relationshipType, between the operational cost and capacity of the nodes. In this experiment, the selection range of the number of nodes, $n$, was 20,50 , and 100 , the selection range of the number of customer clusters, $m$, was 80,100 , and 200 , and the selection range of the relationship type, relationshipType, was linear relationship, concave function relationship, and convex function relationship. There were 27 parameter combinations ( $m, n$, relationshipType), which equated to 27 groups of test cases. The capacity of the nodes, the business of the customer clusters, and the coordinates of the nodes and customer clusters were randomly generated in the range, and were [2000, 3000], [50, 200], and [0, 10000], respectively. Other experimental configuration parameters remained constant. The coverage of the node was 3000, and the unit transportation cost between customer clusters and nodes was 0.01 .

\section{Algorithm Parameters}

The parameters of the SAA included the initial temperature, $t_{0}$, the termination temperature, $t_{\text {end }}$, the cooling coefficient, $a$, and the iteration number, $N$. The performance and quality of the algorithm depended strongly on the parameters. Hence, the parameters had to be adjusted to determine the best combination of algorithm parameters. The cross-comparison method was selected to adjust the parameters using one of the experimental configuration parameter combinations given in the previous section, where $m=20, n=80$, and the relationshipType was linear. The algorithm was run twenty

Table 3. Experimental configuration parameters

\begin{tabular}{|l|l|}
\hline \multicolumn{1}{|c|}{ Parameters } & \\
\hline$n$ & $20,50,100$ \\
\hline$m$ & $80,100,200$ \\
\hline range of the nodes' capacity & {$[2000,3000]$} \\
\hline range of the customer clusters' business & {$[50,200]$} \\
\hline range of the coordinate & {$[0,10000]$} \\
\hline coverage of the nodes & 3000 \\
\hline unit transportation cost & 0.01 \\
\hline relationshipType & linear, concave function, convex function \\
\hline
\end{tabular}


times for every combination of algorithm parameters, and the average of the objective function was taken for comparison.

\section{Initial Temperature, $t_{0}$}

In order to avoid falling into a local minimum, the initial temperature, $t_{0}$, should be set as large as possible. The variables were fixed to $N=300, t_{\text {end }}=1$, and $a=0.9$, while $t_{0}$ was gradually adjusted by increments of 10. For different value of $t_{0}$, the program was run 20 times, the average of the objective function was recorded, and the average runtime of the program was recorded. The left hand of Figure 9 displays the line chart showing the change in the average objective function and average runtime with $t_{0}$. It can be seen from this figure that the average of the objective function decreased and the average runtime increased with an increase in $t_{0}$. When $t_{0}$ exceeded 600, the value of the objective function did not experience a significant change.

\section{Termination Temperature, $t_{\text {end }}$}

The termination temperature, $t_{\text {end }}$, was the temperature of the termination state. The lower $t_{\text {end }}$ was, the more likely the algorithm was to obtain the global optimal solution. This condition also increased the runtime of the program. The variables were fixed to $N=300, t_{0}=400$, and $a=0.9$, while $t_{\text {end }}$ changed gradually. For different values of $t_{\text {end }}$, the program was run 20 times, the average of the objective function was recorded, and the average runtime of the program was recorded. The center hand of Figure 9 displays the line chart showing the change in the average objective function and average runtime with $t_{\text {end }}$. It can be seen from this figure that the average of the objective function and average runtime increased with an increase in $t_{\text {end }}$.

According to the experiments for $t_{0}$ and $t_{\text {end }}$, it can be concluded that a better solution was obtained when a higher ratio of $t_{0}$ to $t_{\text {end }}$ was set. However, this was obtained at the expense of runtime. To balance quality of the solution with runtime of the program, the variables were set to $t_{0}=400$ and $t_{\text {end }}=0.1$.

\section{Cooling Coefficient, a}

The cooling function selected in this study was $T_{k}=t_{0} * a^{k}$, and the final value of $T_{k}$ was $t_{\text {end }}$. The cooling coefficient, $a$, could control the speed of the cooling process. When the cooling process was slow enough, the algorithm could converge on the global optimal solution. However, the value of $a$ must not be too high, otherwise the algorithm would stabilize too early, the optimal solution would not be found, and the runtime would be greatly increased. The variables were fixed to $t_{\text {end }}=0.1, N=300$, and $t_{0}=400$, while $a$ was changed gradually. The right hand of Figure 9 displays the line chart showing the change in the average objective function and average runtime with $a$. It can be seen from this figure that when $a$ exceeds 0.9 , the change in the objective function tends to be stable. When $a=0.97$, the objective function increased instead of decreasing. At the same time, the runtime increased with an increase in $a$. Therefore, $a$ was set to 0.9 .

\section{Iteration Number, $N$}

The greater the iteration number, $N$, was, the more likely it was to obtain the global optimal solution. However, if the algorithm iterated too many times, the runtime of the algorithm increased. Therefore, a reasonable number of iterations had to be determined. The variables were fixed to $t_{\text {end }}=0.1, a=0.9$, and $t_{0}=400$, while $N$ changed gradually in increments of 50. The left side of Figure 10 displays the line chart showing the change in the average objective function and average runtime with $N$. The right side of Figure 10 displays the line chart of the optimal objective function solution after running the program 20 times with various values of $N$. It can be seen from Figure 10 that when $N$ exceeded 300, the change in the objective function tended to be stable. The more iterations that were run through, the easier it was to find the global optimal solution. However, the number of iterations should not be too high, as it significantly increased the runtime of the program. Finally, the number of iterations for each temperature was set to 300 . 
Table 4. Parameters of the three algorithms

\begin{tabular}{|l|l|l|l|}
\hline \multicolumn{1}{|c|}{ Parameters } & \multicolumn{1}{|c|}{ SA } & \multicolumn{1}{c|}{ DIGA } & \multicolumn{1}{c|}{ UIGA } \\
\hline The initial temperature $t_{0}$ & 400 & - & - \\
\hline The termination temperature $t_{\text {end }}$ & 0.1 & - & - \\
\hline The cooling coefficient $a$ & 0.9 & - & - \\
\hline The iteration number $N$ & 300 & 50 & 100 \\
\hline The cancel parameter $-p_{a}$ & 0.3 & - & - \\
\hline The exchange probability $-p_{b}$ & 0.5 & - & - \\
\hline The moving out probability $-p_{c}$ & 0.4 & - & - \\
\hline The utilization rate threshold $-p_{d}$ & 0.3 & - & - \\
\hline The crossover probability $-p c$ & - & 0.5 & 0.5 \\
\hline The mutation probability $-p m$ & - & 0.4 & 0.4 \\
\hline The select parameter $-s l$ & - & 0.95 & 0.95 \\
\hline The antibody number $-p l$ & - & 200 & 200 \\
\hline
\end{tabular}

Figure 9. Change in the average cost and average runtime with the $t_{0}, t_{\text {end }}$ and $a$.
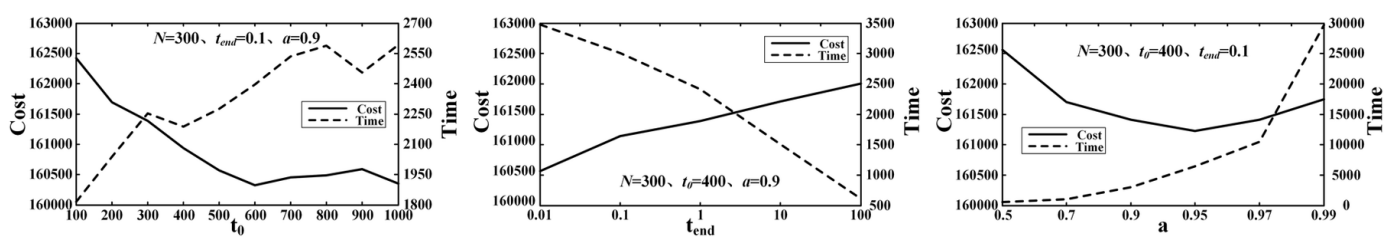

Figure 10. Change in average cost, average runtime, and minimum cost with the number of iterations.
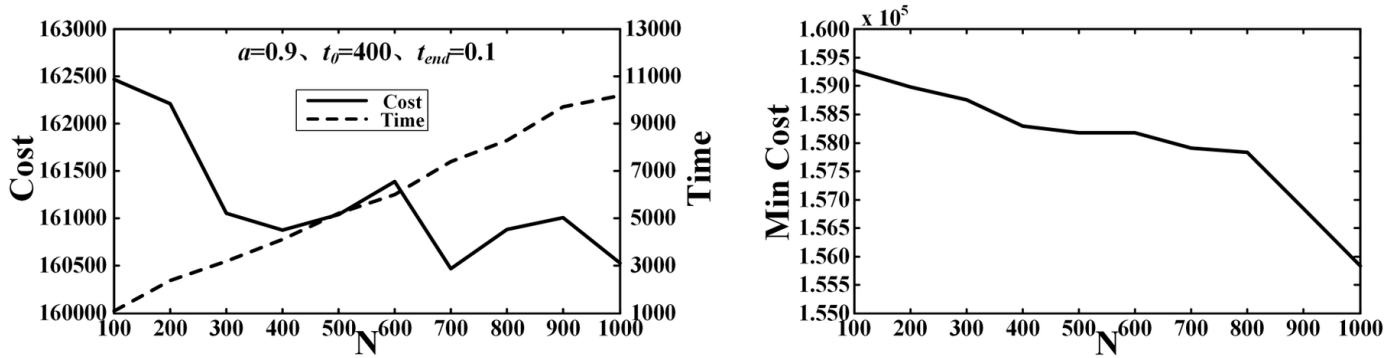

The cross-comparison method was used to adjust other parameters in the SAA, as well as the parameters of the UIGA and DIGA. These efforts are not discussed here owing to limited space. All of the parameters for the three algorithms are shown in Table 4.

\section{Experiments and Results}

\section{Comparison of The Four Solution Methods}

In the experiments, 27 sets of data with different configurations were set up, and four solutions were implemented to solve the problem. Each experimental data set was run 20 times. The experimental 
Figure 11. Average cost and runtime with the three relationships
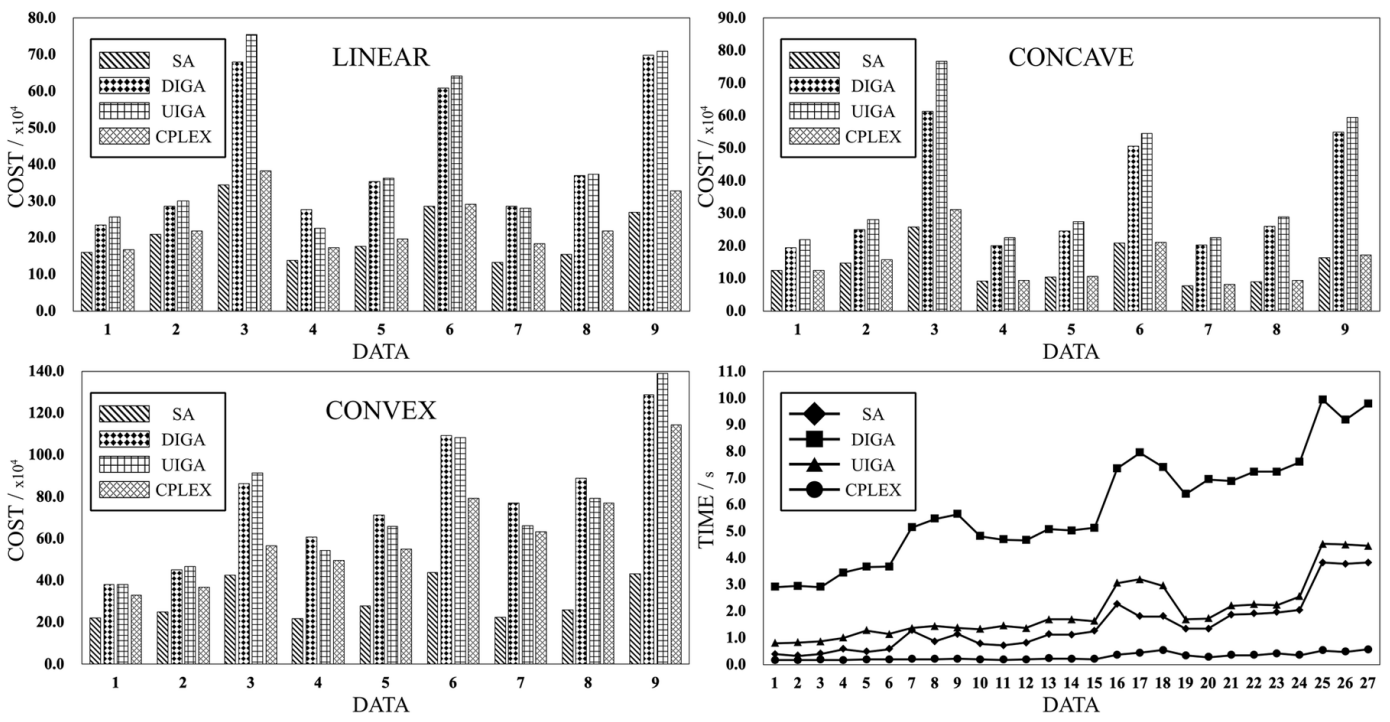

results of the three algorithms and CPLEX solver were compared according to three indexes: cost, average utilization rate of the nodes (ANUR), and the number of nodes to be canceled (NRN). The cost represents the objective function as Eq. (2), and the lower the cost is, the better the distribution scheme is. The ANUR represents the average utilization rate of all the nodes which were not canceled. The higher the ANUR is, the better the distribution scheme is. The formula to calculate the ANUR is given by:

$A N U R=\frac{\sum_{j=1}^{n} \frac{\text { nodeOccupy }_{j}}{f_{j}}}{n-N R N}$

where the number of revocation nodes $(\mathrm{NRN})$ represents the number of nodes which were canceled. The larger the $N R N$, the better the distribution scheme is. $N R N$ is defined as follows:

$N R N=\sum_{j=1}^{n} y_{j}$

Table 5 shows the average cost, minimum cost, and average running time of each data set after running the program 20 times. The cost and running time of the CPLEX solver is also shown in the table. The average costs and the minimum costs of SA are the lowest with all 27 sets of data, however, the average running times of CPLEX are the lowest.

In order to make the simulation data closer to the real situation, the experiments set three relationship types (relationshipType), between the operational cost and capacity of the nodes. The relationship types include linear, concave function and convex function. The Figure 11 shows the average cost of the four solutions. The first figure shows the average cost of the four solutions when the relationship type is linear, and the relationship type of the second one is concave function, and the third one is convex function. It can be seen from the figures that, whatever the relationship type is, the objective function value of the SAA is not only better than the UIGA and DIGA, but also better than 
Table 5. Average cost, minimum cost, and average runtime for the four solution methods

\begin{tabular}{|c|c|c|c|c|c|c|c|c|c|c|c|}
\hline \multirow[b]{2}{*}{ DATA } & \multicolumn{3}{|c|}{ SA } & \multicolumn{3}{|c|}{ IGA } & \multicolumn{3}{|c|}{ UIGA } & \multicolumn{2}{|c|}{ CPLEX } \\
\hline & $\begin{array}{c}\text { AVE } \\
\left(* 10^{4}\right)\end{array}$ & $\begin{array}{c}\text { MIN } \\
\left(* 10^{4}\right)\end{array}$ & $\begin{array}{c}\text { TIME } \\
(/ \mathbf{m s})\end{array}$ & $\begin{array}{c}\text { AVE } \\
\left({ }^{* 10^{4}}\right)\end{array}$ & $\frac{\text { MIN }}{\left(* 10^{4}\right)}$ & $\begin{array}{l}\text { TIME } \\
(/ \mathbf{m s})\end{array}$ & $\begin{array}{c}\text { AVE } \\
\left(* 10^{4-}\right)\end{array}$ & $\frac{\text { MIN }}{\left(* 10^{4-}\right)}$ & $\begin{array}{c}\text { TIME } \\
(/ \mathbf{m s})\end{array}$ & $\begin{array}{l}\text { COST } \\
\left(* 10^{4}\right)\end{array}$ & $\begin{array}{c}\text { TIME } \\
(/ \mathbf{m s})\end{array}$ \\
\hline 1 & $\underline{15.9}$ & $\underline{15.7}$ & 383.4 & 23.4 & 22.7 & 2914.7 & 25.5 & 23.9 & 800.0 & 16.7 & $\underline{161.0}$ \\
\hline 2 & $\underline{12.4}$ & $\underline{12.4}$ & 322.6 & 19.4 & 19.0 & 2950.8 & 21.9 & 20.8 & 823.1 & 12.5 & $\underline{159.0}$ \\
\hline 3 & 21.9 & $\underline{20.5}$ & 403.3 & 38.0 & 37.2 & 2906.1 & 38.1 & 35.9 & 860.0 & 33.0 & $\underline{175.0}$ \\
\hline 4 & $\underline{20.9}$ & $\underline{20.2}$ & 570.6 & 28.5 & 27.8 & 3453.0 & 30.0 & 28.9 & 1003.2 & 21.8 & $\underline{164.0}$ \\
\hline 5 & $\underline{14.6}$ & $\underline{14.5}$ & 469.6 & 24.8 & 24.3 & 3658.5 & 28.0 & 26.7 & 1283.2 & 15.6 & $\underline{180.0}$ \\
\hline 6 & $\underline{25.0}$ & $\underline{23.0}$ & 574.6 & 45.1 & 44.1 & 3674.4 & 46.6 & 44.7 & 1134.7 & 36.8 & 186.0 \\
\hline 7 & $\underline{34.3}$ & $\underline{33.1}$ & 2278.6 & 67.9 & 67.9 & 8146.0 & 75.4 & 71.8 & 1366.7 & 38.2 & 196.0 \\
\hline 8 & $\underline{25.7}$ & $\underline{24.7}$ & 1866.2 & 61.3 & 60.7 & 8468.4 & 76.8 & 73.5 & 1445.6 & 31.1 & $\underline{201.0}$ \\
\hline 9 & 42.4 & $\underline{38.4}$ & 2143.4 & 86.3 & 85.5 & 8646.0 & 91.3 & 88.5 & 1384.0 & 56.5 & $\underline{207.0}$ \\
\hline 10 & $\underline{13.7}$ & $\underline{13.5}$ & 778.0 & 27.7 & 27.0 & 5821.7 & 22.5 & 21.5 & 1322.8 & 17.2 & 188.0 \\
\hline 11 & 9.1 & 9.17 & 711.8 & 20.0 & 19.2 & 5687.1 & 22.4 & 21.1 & 1456.2 & 9.4 & $\underline{176.0}$ \\
\hline 12 & $\underline{21.8}$ & $\underline{21.3}$ & 816.6 & 60.7 & 59.4 & 5662.7 & 54.3 & 50.8 & 1363.6 & 49.6 & $\underline{182.0}$ \\
\hline 13 & $\underline{17.6}$ & $\underline{16.9}$ & 1122.8 & 35.3 & 34.6 & 7078.0 & 36.2 & 34.0 & 1697.1 & 19.7 & $\underline{223.0}$ \\
\hline 14 & $\underline{10.4}$ & $\underline{10.3}$ & 1108.6 & 24.6 & 24.0 & 7025.1 & 27.3 & 25.7 & 1686.8 & 10.6 & $\underline{209.0}$ \\
\hline 15 & $\underline{27.7}$ & $\underline{26.2}$ & 1250.9 & 71.2 & 69.3 & 8128.4 & 65.7 & 60.5 & 1624.7 & 54.8 & $\underline{198.0}$ \\
\hline 16 & $\underline{28.6}$ & $\underline{28.0}$ & 4265.3 & 60.9 & 59.6 & 15360.4 & 64.2 & 60.7 & 3063.6 & 29.2 & $\underline{357.0}$ \\
\hline 17 & $\underline{20.8}$ & $\underline{20.8}$ & 3810.3 & 50.6 & 49.8 & 16957.1 & 54.5 & 52.0 & 3199.6 & 21.0 & $\underline{437.0}$ \\
\hline 18 & 43.9 & $\underline{42.0}$ & 3810.3 & 109.4 & 107.5 & 16410.4 & 108.4 & 105.1 & 2958.7 & 79.1 & $\underline{532.0}$ \\
\hline 19 & $\underline{13.2}$ & $\underline{13.0}$ & 1338.4 & 28.5 & 28.0 & 10398.4 & 27.9 & 26.5 & 1687.5 & 18.3 & 338.0 \\
\hline 20 & $\underline{7.7}$ & $\underline{7.7}$ & 1335.3 & 20.3 & 19.8 & 9946.6 & 22.5 & 21.2 & 1734.9 & 8.1 & $\underline{275.0}$ \\
\hline 21 & $\underline{22.5}$ & $\underline{21.0}$ & 1867.1 & 77.0 & 75.4 & 9880.8 & 66.1 & 62.7 & 1803.6 & 63.3 & 350.0 \\
\hline 22 & $\underline{15.5}$ & 15.4 & 1908.9 & 37.0 & 35.8 & 13240.0 & 37.2 & 35.5 & 2253.8 & 21.8 & 355.0 \\
\hline 23 & $\underline{8.8}$ & $\underline{8.8}$ & 1961.0 & 25.9 & 25.2 & 13232.5 & 28.8 & 27.0 & 2237.0 & 9.3 & $\underline{408.0}$ \\
\hline 24 & $\underline{25.7}$ & $\underline{24.7}$ & 2140.1 & 88.9 & 85.9 & 13595.7 & 79.1 & 72.5 & 2159.3 & 76.9 & $\underline{348.0}$ \\
\hline 25 & $\underline{26.9}$ & $\underline{26.4}$ & 3828.8 & 69.7 & 68.4 & 16950.2 & 70.8 & 67.9 & 4524.0 & 32.8 & $\underline{531.0}$ \\
\hline 26 & $\underline{16.4}$ & $\underline{16.4}$ & 3771.7 & 55.0 & 53.9 & 16179.3 & 59.4 & 57.4 & 4504.7 & 17.1 & $\underline{470.0}$ \\
\hline 27 & $\underline{43.3}$ & $\underline{42.1}$ & 3824.1 & 28.6 & 149.2 & 16790.1 & 140.1 & 144.8 & 4457.2 & 114.2 & $\underline{558.0}$ \\
\hline
\end{tabular}

the CPLEX solver. The fourth picture of Figure 11 shows a comparison of the average running times of the three algorithms and CPLEX solver. It can be seen from the figure that the running time of the SAA was longer than that of the CPLEX solver, but shorter than the UIGA and DIGA. Therefore, in the above three cases, the SAA performed better than the other three methods.

Figure 12 shows the comparison of the ANUR and $N R N$ for the four methods, respectively. The higher the ANUR is, the better the distribution scheme is. Additionally, the larger the $N R N$ is, the better the distribution scheme is. It can be seen from the two figures that, when using the SAA to solve this problem, the ANUR and NRN is higher than other three methods, so the distribution schemes solved by SAA is better than the schemes solved by other three methods. 

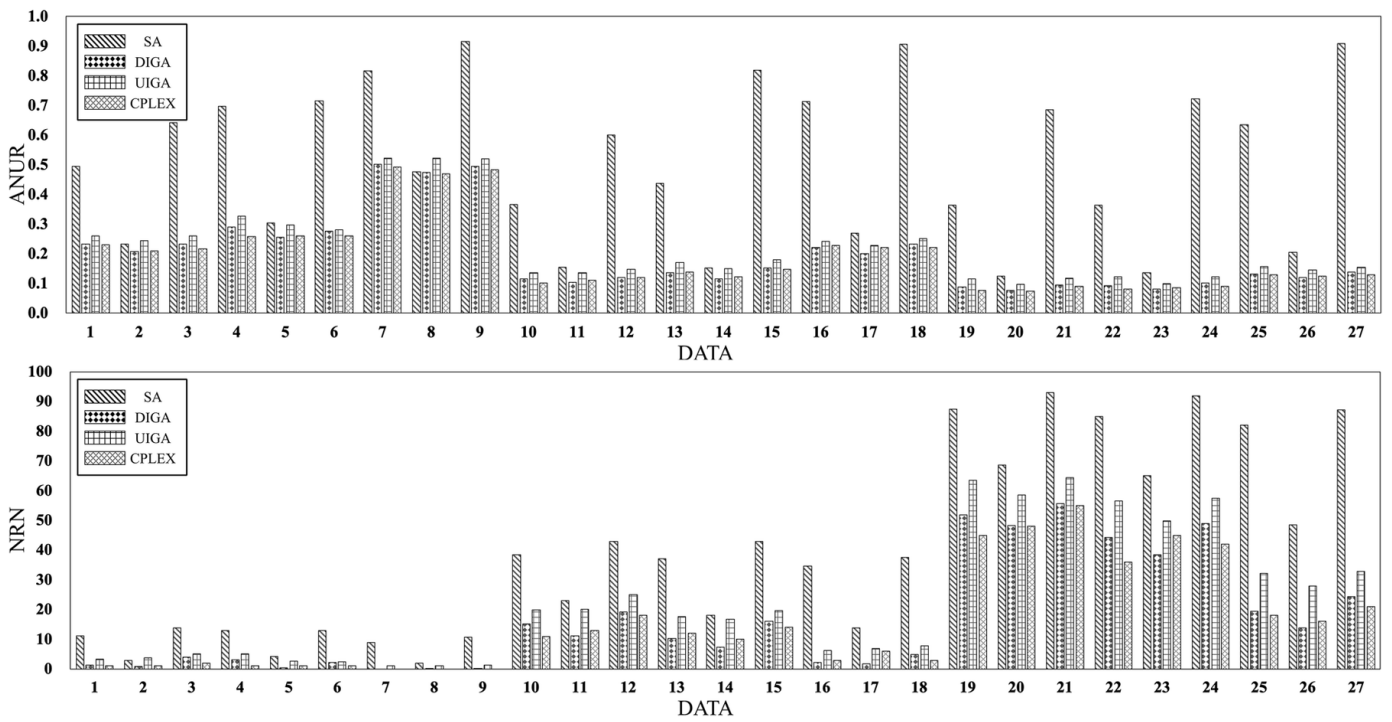

\section{Comparison of the Relationships Between the Operational Cost and Capacity of the Nodes}

In the experiments designed for this study, there were three types of relationships between the operational cost and capacity of the nodes, including linear, concave function, and convex function. In order to find which situation this method is more suitable for, experiments were designed to see the ANUR and NRN of the SAA in three cases with different relationship types. The result is shown in Figure 13. For example, the first set of data were ran with three different relationship types, and the other parameters were fixed as $n=20$ and $m=80$. It can be seen from the figures that when the relationship was the convex function, the ANUR was higher and the $N R N$ was also higher, which means the model and algorithm designed for this study were more suitable for the situation where the relationship was the convex function.

\section{Relationship Between the ANUR and $M$ and $N$}

In the experiments designed for this study, the selection range for $n$ was 20,50 , and 100 , and the selection range for $m$ was 80,100, and 200. Table 6 shows the value of the ANUR with various $m$ and $n$ values when the relationship was linear. It can be seen from the Table 6 that, when fixing $n$, the ANUR increased with $m$. While fixing $m$, the ANUR decreased with $n$. This indicated that the

Figure 13. ANUR and NRN comparison for the SAA in three cases
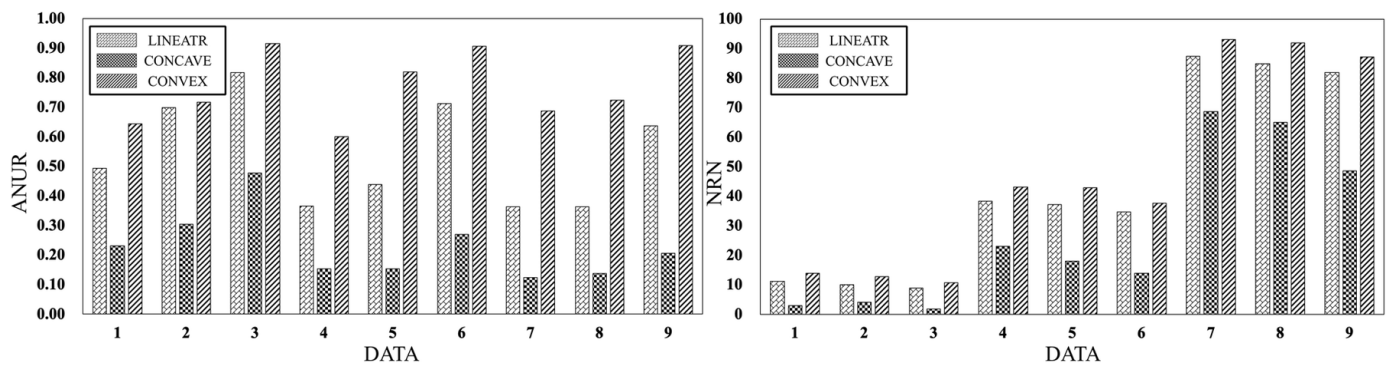
Table 6. Relationship between the ANUR and $\mathrm{m}$ and $\mathrm{n}$ for the three cases

\begin{tabular}{|l|l|l|l|l|l|l|l|l|l|l|l|l|}
\hline \multicolumn{4}{|c|}{ linear relationship } & \multicolumn{3}{c|}{ concave function relationship } & \multicolumn{3}{c|}{ convex function relationship } \\
\hline & $m=\mathbf{8 0}$ & $m=\mathbf{1 0 0}$ & $m=\mathbf{2 0 0}$ & & $m=\mathbf{8 0}$ & $m=\mathbf{1 0 0}$ & $m=\mathbf{2 0 0}$ & & $m=\mathbf{8 0}$ & $m=\mathbf{1 0 0}$ & $m=\mathbf{2 0 0}$ \\
\hline $\boldsymbol{n = 2 0}$ & 0.49 & 0.70 & 0.82 & $\boldsymbol{n = 2 0}$ & 0.23 & 0.30 & 0.48 & $\boldsymbol{n = 2 0}$ & 0.64 & 0.72 & 0.91 \\
\hline $\boldsymbol{n = 5 0}$ & 0.37 & 0.44 & 0.71 & $\boldsymbol{n = 5 0}$ & 0.15 & 0.16 & 0.27 & $\boldsymbol{n = 5 0}$ & 0.60 & 0.62 & 0.84 \\
\hline $\boldsymbol{n = 1 0 0}$ & 0.36 & 0.36 & 0.64 & $\boldsymbol{n = 1 0 0}$ & 0.12 & 0.14 & 0.21 & $\boldsymbol{n = 1 0 0}$ & 0.49 & 0.52 & 0.81 \\
\hline
\end{tabular}

$A N U R$ was inversely proportional to the ratio of $n$ to $m$. Therefore, it was concluded that the model and algorithm designed for this study were more suitable for the situation where the number of nodes was higher and the number of customer clusters was lower, indicating that the supply of business provided by the nodes was far greater than the demand of business required by the customer clusters.

\section{Comparison of The Four Neighborhood Operations}

The algorithm proposed in this study utilized four neighborhood operations. In order to verify every operation is effective in solving the ETNOIP, an experiment was designed. Removed one operation, and the results gathered by running algorithm with other three operations were compared with the results of original algorithm with all four operations. There were five combinations including here \{rule $1+2+3+4$ (original algorithm), rule $2+3+4$ (remove rule 1 ), rule $1+3+4$ (remove rule 2 ), rule $1+2+4$ (remove rule 3 ), rule $1+2+3$ (remove rule 4 ) $\}$. Figure 14 shows the comparative result. The lower the cost is, the better the distribution scheme is. It can be seen that the cost gathered by original algorithm is the lowest, indicated that no matter which operation is removed, the performance of algorithm will be reduced. That is to say, every operation was effective in solving the optimization problem. The result was worst when rule 1 was removed, and only rules 2,3 , and 4 were used. Therefore, rule 1 playes the most significant role in the optimization process.

The experiments designed for this study verified the effectiveness of the SAA in solving the optimization problem in four parts. Through the above comparative experiments, it can be concluded that:

1. Compared with the other three methods, the cost was lower, the utilization rate higher, and the integration effect better when the ETNOIP was solved by the SAA. When using UIGA and DIGA to solve it, the runtime was extremely long, and the result was worse than using the SAA. Although the SAA was not as fast as the CPLEX solver, the result was better.

2. The relationship between the operational cost and the capacity of the nodes was comprised of three types: a linear relationship, a concave function relationship, and a convex function relationship.

Figure 14. Difference between using any three rules to solve the problem vs. using all four rules

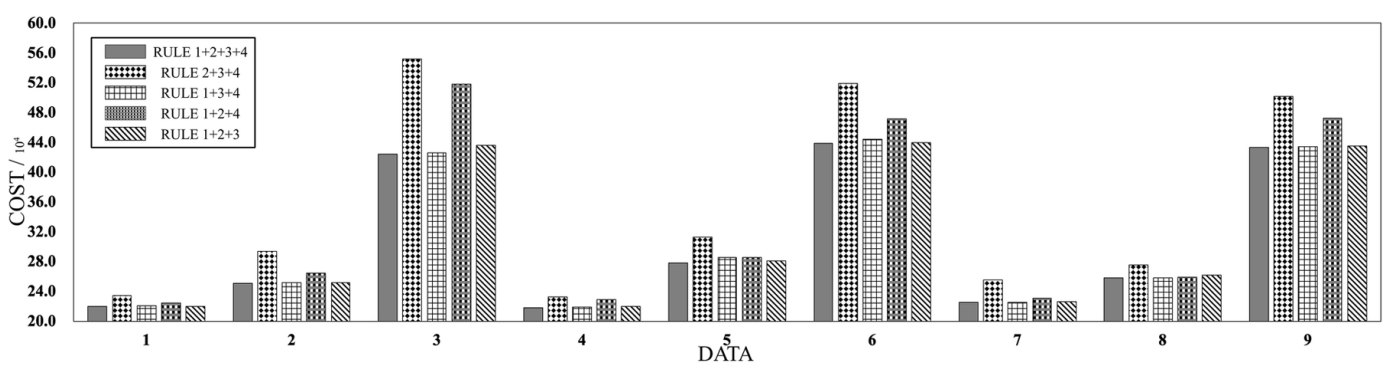


Compared with the experimental results, it can be seen that the model and algorithm designed for this study were more suitable for situations when the relationship was convex,

3. The average utilization rate of the nodes was inversely proportional to the ratio of the number of nodes to the number customer clusters. The model and the algorithm designed for this study were more suitable for situations with more nodes and fewer customer clusters. That is, the supply business provided by the nodes was far greater than the demand business required by the customer clusters.

4. Four neighborhood operation rules were proposed, and experiments were designed to verify their effectiveness. It was concluded that rule 1 played an important role in the process of solving the ETNOIP.

\section{CONCLUSION}

There are deficiencies in the existing methods used to solve the ETNOIP. In view of these shortcomings, this study proposed an express terminal nodes optimization and integration model with detachable business for single customer clusters in order to improve the utilization rate of the network, reduce the operational cost of the nodes, and reduce the transportation cost between the nodes and customer clusters. The objective function of the model was to minimize the total cost. Additionally, improvements to the SAA were achieved based on a neighborhood search to solve the problem. To test and verify the effectiveness of the SAA in the optimization process, the UIGA, DIGA, and CPLEX solver were utilized as comparison experiments. The results demonstrated that the cost was lower, the utilization rate was higher, and the integration effect was better when solving the ETNOIP using the SAA. It can also be seen from the results that the model and algorithm designed for this study were more suitable for situations where the supply of business provided by the nodes is far greater than the demand of business required by the customer clusters. Four neighborhood rules were designed and implemented to further verify their effectiveness. It was concluded that every neighborhood rule played a role in the optimization process, although rule 1 played a more significant role.

There are still some shortcomings in this study. The objective function was not comprehensive enough, for example, it did not consider the two-level distribution with transit center nodes, nodes, and customer clusters. In the algorithm implementation, when compared with the CPLEX solver, the runtime of the algorithm was slower, the solution results were not fixed, and their quality degraded with every run of the program. Further improvements and optimizations are needed. 


\section{ACKNOWLEDGMENT}

This research was supported by the Key R\&D Plan Project of China [grant number 2018YFB1403104]; and the Key R\&D Plan Project of Shandong Province, China [grant number GG201703130116].

\section{REFERENCES}

Droździel, P., Wińska, M., Madleňák, R., \& Szumski, P. (2017). Optimization of the post logistics network and location of the local distribution center in selected area of the Lublin province. Procedia Engineering, 192, 130-135. doi:10.1016/j.proeng.2017.06.023

Duan, H., Song, G., Qu, S., Dong, X., \& Xu, M. (2019). Post-consumer packaging waste from express delivery in China. Resources, Conservation and Recycling, 144, 137-143. doi:10.1016/j.resconrec.2019.01.037

Guastaroba, G., \& Speranza, M. G. (2014). A heuristic for BILP problems: The single source capacitated facility location problem. European Journal of Operational Research, 238(2), 438-450. doi:10.1016/j.ejor.2014.04.007

Hakimi, S. L. (1964). Optimum locations of switching centers and the absolute centers and medians of a graph. Operations Research, 12(3), 450-459. doi:10.1287/opre.12.3.450

Hazlewood, W. R., \& Coyle, L. (2009). On ambient information systems: challenges of design and evaluation. International Journal of Ambient Computing \& Intelligence, 1(2), 1-12.

He, Y., Wang, X., Lin, Y., Zhou, F., \& Zhou, L. (2017). Sustainable decision making for joint distribution center location choice. Transportation Research Part D, Transport and Environment, 55, 202-216. doi:10.1016/j. $\operatorname{trd} .2017 .07 .001$

Hua, X., Hu, X., \& Yuan, W. (2016). Research optimization on logistics distribution center location based on adaptive particle swarm algorithm. Optik (Stuttgart), 127(20), 8443-8450. doi:10.1016/j.ijleo.2016.06.032

Jaramillo, J. H., Bhadury, J., \& Batta, R. (2002). On the use of genetic algorithms to solve location problems. Computers \& Operations Research, 29(6), 761-779. doi:10.1016/S0305-0548(01)00021-1

Ji, Y., Yang, H., Zhang, Y., \& Zhong, W. (2013). Location optimization model of regional express distribution center. Procedia: Social and Behavioral Sciences, 96, 1008-1013. doi:10.1016/j.sbspro.2013.08.115

Joerss, M., Schröder, J., Neuhaus, F., Klink, C., \& Mann, F. (2016). Parcel delivery: The future of last mile. McKinsey \& Company.

Kirkpatrick, S., Gelatt, C. D., \& Vecchi, M. P. (1983). Optimization by simulated annealing. Science, 220(4598), 671-680.

Klose, A., \& Görtz, S. (2007). A branch-and-price algorithm for the capacitated facility location problem. European Journal of Operational Research, 179(3), 1109-1125. doi:10.1016/j.ejor.2005.03.078

Lai, M. C., Sohn, H. S., Tseng, T. L. B., \& Chiang, C. (2010). A hybrid algorithm for capacitated plant location problem. Expert Systems with Applications, 37(12), 8599-8605. doi:10.1016/j.eswa.2010.06.104

Liu, Y., Fan, L., Wang, Y., \& Dong, Q. (2009, December). Optimization of Logistics Nodes in Dynamic Location with a Multi-objective Evolutionary Algorithm. In 2009 International Conference on Computational Intelligence and Security (Vol. 1, pp. 133-137). IEEE. doi:10.1109/CIS.2009.101

Melkote, S., \& Daskin, M. S. (2001). Capacitated facility location/network design problems. European Journal of Operational Research, 129(3), 481-495. doi:10.1016/S0377-2217(99)00464-6

Nozick, L. K. (2001). The fixed charge facility location problem with coverage restrictions. Transportation Research Part E, Logistics and Transportation Review, 37(4), 281-296. doi:10.1016/S1366-5545(00)00018-1

Pal, M., Tardos, T., \& Wexler, T. (2001, October). Facility location with nonuniform hard capacities. In Proceedings 42nd IEEE Symposium on Foundations of Computer Science (pp. 329-338). IEEE. doi:10.1109/ SFCS.2001.959907 
Sun, H., Gao, Z., \& Wu, J. (2008). A bi-level programming model and solution algorithm for the location of logistics distribution centers. Applied Mathematical Modelling, 32(4), 610-616. doi:10.1016/j.apm.2007.02.007

Sun, L., Karwan, M. H., Gemici-Ozkan, B., \& Pinto, J. M. (2015). Estimating the long-term cost to serve new customers in joint distribution. Computers \& Industrial Engineering, 80, 1-11. doi:10.1016/j.cie.2014.11.012

Ting, C. J., \& Chen, C. H. (2013). A multiple ant colony optimization algorithm for the capacitated location routing problem. International Journal of Production Economics, 141(1), 34-44. doi:10.1016/j.ijpe.2012.06.011

Venables, H., \& Moscardini, A. (2006, September). An adaptive search heuristic for the capacitated fixed charge location problem. In International Workshop on Ant Colony Optimization and Swarm Intelligence (pp. 348-355). Springer. doi:10.1007/11839088_32

Venables, H., \& Moscardini, A. (2008, September). Ant based heuristics for the capacitated fixed charge location problem. In International Conference on Ant Colony Optimization and Swarm Intelligence (pp. 235-242). Springer. doi:10.1007/978-3-540-87527-7_22

Wang, W., Rahman, M. A., Kurniawan, I. F., Asyhari, A. T., Sadat, S. N., \& Yao, L. (2019, February). Immune Genetic Algorithm Optimization and Integration of Logistics Network Terminal Resources. In 2019 Third IEEE International Conference on Robotic Computing (IRC) (pp. 435-436). IEEE.

Wang, Y., Ma, X., Liu, M., Gong, K., Liu, Y., Xu, M., \& Wang, Y. (2017). Cooperation and profit allocation in two-echelon logistics joint distribution network optimization. Applied Soft Computing, 56, 143-157. doi:10.1016/j. asoc. 2017.02 .025

Wu, L. Y., Zhang, X. S., \& Zhang, J. L. (2006). Capacitated facility location problem with general setup cost. Computers \& Operations Research, 33(5), 1226-1241. doi:10.1016/j.cor.2004.09.012

Xu, Y., Xu, D., Du, D., \& Zhang, D. (2019). Approximation algorithm for squared metric facility location problem with nonuniform capacities. Discrete Applied Mathematics, 264, 208-217. doi:10.1016/j.dam.2019.03.013

Yamin, M., \& Sen, A. A. A. (2018). Improving privacy and security of user data in location based services. International Journal of Ambient Computing and Intelligence, 9(1), 19-42. doi:10.4018/IJACI.2018010102

Yang, W., Wang, X., Song, X., Yang, Y., \& Patnaik, S. (2018). Design of intelligent transportation system supported by new generation wireless communication technology. International Journal of Ambient Computing and Intelligence, 9(1), 78-94. doi:10.4018/IJACI.2018010105

Zhang, J., Chen, B., \& Ye, Y. (2005). A multiexchange local search algorithm for the capacitated facility location problem. Mathematics of Operations Research, 30(2), 389-403. doi:10.1287/moor.1040.0125

Fanchao Meng is an Associate Professor in the School of Computer Science and Technology, Harbin Institute of Technology at Weiahi, China. He holds a Ph.D. degree in Computer Application Technology from Harbin Institute of Technology. His research interests are cloud computing, Combinatorial optimization, Enterprise resource planning and logistics management.

Qingran Ji is studying for M.S. degree in the School of Computer Science and Technology, Harbin Institute of Technology at Weiahi, China.

Zheng Hongzhen, doctor, professor, provincial teacher, research fields: supply chain management and modern logistics, data mining, enterprise intelligence and business intelligence. She is the teaching leader of Harbin Institute of Technology and a member of the national computer education research association. Won the second prize of provincial science and technology progress, the second prize of provincial computer application technology outstanding achievement, the first prize of municipal science and technology progress, etc. Among them, the provincial graduate student innovation research project has won the first prize of the university's teaching achievements, more than 20 software Copyrights, published more than 60 papers in major publications at home and abroad, including more than 10 teaching and research papers, and edited 2 textbooks.

Huihui Wang has studied in School of Computer Science and Technology, Harbin Institute of Technology at Weihai, Weihai, China.

Dianhui Chu, Ph.D., Professor, doctoral supervisor, Dean of School of Computer Science and Technology, Harbin Institute of Technology, Weihai, Executive Deputy Director of Shandong Enterprise Network and E-business Engineering Technology Research Center, and Director of Weihai Health Care Service Engineering Research Center. He mainly engages in the work of service computing and software services engineering, business intelligence and data mining, health care services technology, etc. 